\title{
APORTACIONES AL CONOCIMIENTO DE LAS ALGAS DEL SE DE ESPAÑA. IV. LAS DIATOMEAS (BACILLARIOPHYCEAE)
}

\author{
Marina ABOAL
}

RESUMEN: Se presentan un total de 148 táxones de Diatomeas distribuidas por todo tipo de ambientes del SE español. Se aportan datos sobre su ecología y se sumariza por medio de tablas los rangos de variación de los principales factores físico-químicos para la mayoría de las especies.

Palabras clave: Algas dulciacuícolas, Bacillariophyceae, España;

ABSCTRACT: One hundred and forty eight Diatom taxa from Southeastern Spain are reported. Data on ecology and distribution are presented. The range of variation of main physichal-chemical factors for the majority of the species is summarized.

Key words: Freshwater algae, Bacillariophyceae, Spain.

\section{IN TRODUCCION}

Dada la escasez de datos referentes a las algas dulciacuícolas y de aguas salobres que proliferan en los sistemas acuáticos del sureste español, se presentan aquí algunos datos morfológicos, ecológicos y de distribución de Diatomeas, al objeto de completar nuestro conocimiento sobre este grupo que ya había sido tratado con anterioridad aunque de manera muy puntual por Margalef et al. 1977, Aviles et al. 1973 y 1980, Tomás, 1981 y Aboal, 1987, 1988.

\section{AREA DE ESTUDIO}

La zona estudiada es la Cuenca del río Segura (Figura 1) que comprende parte de las provincias de Jaén, Granada, Albacete, Murcia y Alicante. Dentro de este marco geográfico se han prospectado todo tipo de sistemas y ambientes: fuentes, ramblas o arroyos salobres, sistemas con influencia marina y arroyos de agua dulce más o menos permanentes. 


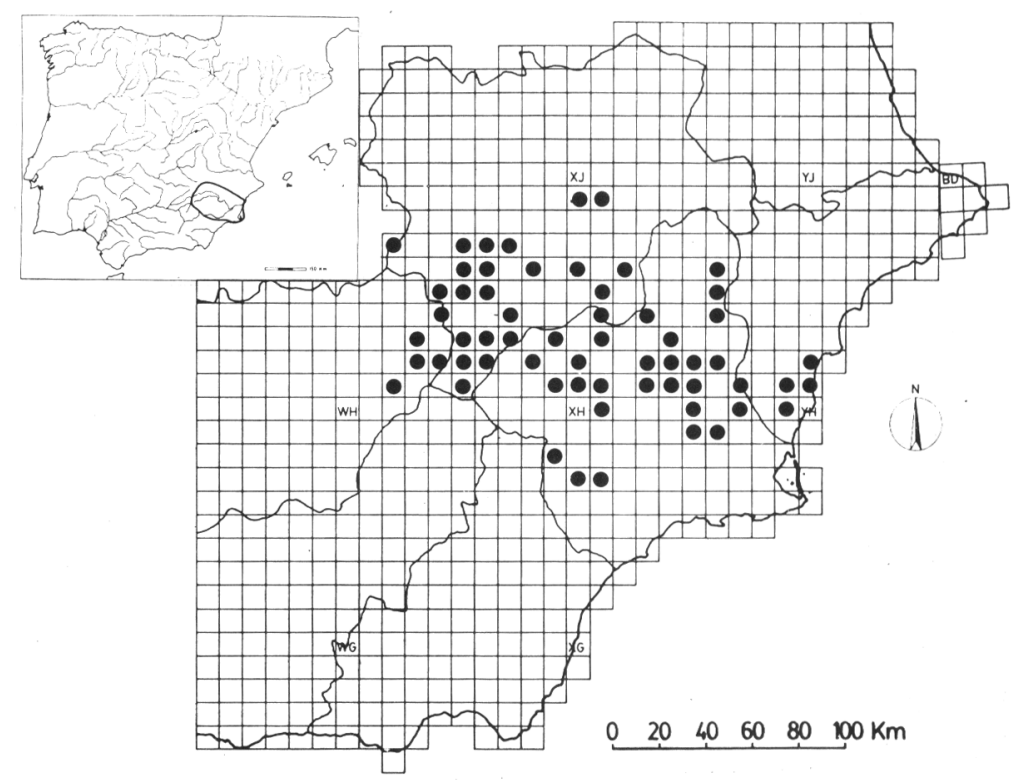

FIGURA 1. Situación geográfica del área de estudio y localización de las estaciones de muestreo en la cuadrícula U.T.M. de diez kilómetros de lado.

Toda la cuenca discurre por terrenos carbonatados que sólo se interrumpen con pequeñas manchas de margas del Neógeno o del Keuper, sobre las que fluyen arroyos o ramblas salinas.

En la Tabla I reunimos todas las localidades consideradas con indicación de coordenadas UTM.

\section{MATERIALES Y METODOS}

Las recolecciones se realizaron en todas las sinusias existentes en la estación de muestreo, mediante succión de las primeras capas de limo (herpon) o rascado de rocas (pecton). En el caso de las especies que viven epífitas o retenidas entre plantas de mayor parte, una vez recolectado este material se exprimió y se conservó el extracto resultante.

En todos los casos, se realizó una maceración previa con ácido sulfúrico concentrado con objeto de eliminar las sales, seguida de centrifugados y lavados sucesivos con agua destilada. A continuación se limpian los frústulos añadiendo a la muestra una mezcla de ácido clorhídrico y ácido nítrico. Se calienta a baño maría, sin llegar a ebullición de 30-45 minutos. Tras una serie de lavados y centrifugados se puede proceder a la observación del material.

Para especies poco silicificadas que no resisten este tratamiento se quemó la materia orgánica al fuego con un mechero Bünzen. 
La separación de los grupos taxonómicos mayores se ha hecho según Bold \& Wynne (1985) y la distinción de especies según Germain (1981), Hustedt (1930 y 1930-66), Krammer \& Lange-Bertalot $(1986,1988)$.

\section{RES ULTADOS \\ O R D E N C E N T R A LE S \\ SUBORDEN COSCINODISCINEAE \\ Familia COSCINODISCACEAE}

\section{Melosira arenaria Moore}

Dimensiones: $60 \times 15-20 \mu \mathrm{m}$.

Sobre briófitos, en paredes rezumantes, forma filamentos visibles a simple vista.

Loc: 1 (VII/83).

Melosira juergensii C.A. Agardh

Dimensiones: $20-25 \times 13-18 \mu \mathrm{m}$.

Bentónica, en riberas de arroyos o ramblas de aguas salobres (Tabla II). IV/85)

Loc: 2 (VII/83).- (VII/83).- 4 (VII/83).- 5 (VII/83).- 6 (IV/84).- 7 (IX/84).- 8 (IX/84,

Melosira moniliformis (Müller) Agardh.

Dimensiones: $35-37$ x 39-40 $\mu \mathrm{m}$.

Junto con algas filamentosas en aguas salobres (Tabla II).

Loc.: 3 (IX/84, IV/85).

*var. subglobosa Grunow

De $45 \times 25 \mu \mathrm{m}$.

En las mismas condiciones que la especie.

Melosira varians C.A. Agardh

Dimensiones: $10-20 \times 20-30 \mu \mathrm{m}$.

Forma largos filamentos flotantes, verde pálidos o pardo intensos, en remansos de riberas de ríos de agua dulce. Puede fijarse a objetos sumergidos. Como ya sugería Margalef (1954a) se hace más escasa al aumentar la altitud. Soporta bien la polución orgánica (Tabla II).

Loc.: 9 (IX/85).- 10 (VII/83).- 11 (VIII/85).- 81 (VIII/85).- 13 (VIII/85).- 14 (VIII/85).15 (VII/83).- 16 (VII/83).- 17 (VII/83).- 18 (VII/83).- 19 (VII/83).- 20 (VIII/85).- 21 (VII/83).- 22 (II/85).

('yclotella alvarniensis (Wütrich) Servant-Vildary

Esta diatomea fósil procede de arrastres desde los yacimientos de diatomita miocénicos de Elche de la Sierra (Albacete). Aparece a veces en cantidades considerables. Presenta una forma triangular de vértices redondeados de 13-15 $\mu \mathrm{m}$ de lado.

Loc.: 58 (VIII/83).- 11 (VII/85).- 81 (VIII/85).- 129 (VIII/85).- 14 (VIII/85).- 7 (IV/84).- 4 (IV/84).

Cyclotella kützingiana Thwaites

Células de 25-28 $\mu \mathrm{m}$ de diámetro. Estrías: 18-20 en $10 \mu \mathrm{m}$.

Metafiton de aguas dulces. No soporta la polución (Tabla II).

Loc.: 22 (IX/84, XI/84).- 23 (VI/83).- 24 (VII/83).- 25 (II/82).- 26 (IX/85).- 27(X/85).-

28 (III/84, XII/84).- 29 (III/84, XII/84).- 30 (VI/81).- 31 (V/81, I/82).- 32 (V/82).- 33

(IV/82).- 34 (XI/81).- 35 (IV/84).- 36 (IV/84).

Cyclotella meneghiniana Kützing

Células de $12 \mu \mathrm{m}$ de diámetro y 12 estrías cada $10 \mu \mathrm{m}$.

Metafiton en aguas dulces y salobres. Eurioica. Soporta la polución (Tabla II). 
Loc.: 3 (IV/84).- 4 (IV/84, IV/85).- 5 (IV/84).- 13 (VIII/85).- 14 (VIII/85).- 22 (IV/85).- 26(IV/83, III/84, IV/84).- 27 (IV/83).- 28 (III/84, IX/85).- 29 (III/84).- 37 (VII/85).- 38 (VIII/85).- 39 (VIII/85).- 40 (VIII/83, III/84).- 41 (II/85).

Stephanodiscus dubius (Fricke) Hustedt

De $15-20 \mu \mathrm{m}$ de diámetro, 10 costillas en $10 \mu \mathrm{m}$.

Metafiton. Aguas salobres circulantes. Para Hustedt (1930) es halófila.

Loc.: 7 (VII/82).- 3 (IV/84).- 4 (IV/85).

Stephanodiscus hantzschii Grunow

De 10-20 $\mu \mathrm{m}$ de diámetro, 8-10 costillas en $10 \mu \mathrm{m}$.

Metafiton. Aguas salobres circulantes. Germain (1981) sostiene que no soporta la presencia de sal pero Hustedt (1930) la encuentra también en aguas salobres.

Loc.: 3 (IV/85).

\section{SUBORDEN BIDDULPHIINEAE Familia CHAETOCERACEAE}

Chaetoceros muelleri Lemmermann

De $15-20 \mu \mathrm{m}$ de longitud con eje pervalvar de hasta 3 veces esa longitud. Cistes presentes.

Planctónica en aguas salobres estancadas (Tabla II).

Loc.: 3 (IV/84, XI/84).

Chaetoceros wighamii Brightwell

Células de $30 \times 12 \mu \mathrm{m}$. Cistes espinulosos característicos.

Planctónica en aguas salobres estancadas o remansadas (Tabla II).

Loc.: 3 (IV/84).- 5 (IV/85).

\section{Familia BIDDULPHIACEAE}

Pleurosira laevis Ehrenberg

Células de 80-115 x 65-75 $\mu \mathrm{m}$. Auxósporas en abril.

Compère (1982) demuestra que este es el nombre válido de Biddulphia laevis Ehrenberg.

Sobre otras algas en riberas de arroyos de aguas dulces. En cursos salobres forma grandes masas parduzcas (Tabla II).

Loc.: 3 (IV/84).- 4 (VII/83).- 5 (VII/83, VIII/85).- 6 (IV/84).- 7 (IX/84).- 11 (VIII/85).-

13 (VIII/85).- 38 (VII/83).- 42 (VIII/85).- 43 (VI/83).- 44 (XII/82).- 45 (VII/83).- 46 (IX/83).- 47 (IX/83).- 48 (VIII/85).- 49 (VII/83).- 50 (VIII/85).- 51 (VII/83).- 52 (III/81).53 (IV/84).- 54 (XI/84).

Biddulphia subaequa (Kützing) Ralfs

De $80-100 \times 60-70 \mu \mathrm{m}$.

Epífita sobre algas filamentosas en aguas circulantes salobres.

Loc.: 7 (VII/82).- 3 (IV/85).

Coscinodiscus radiatus Ehrenberg

De $20-50 \mu \mathrm{m}$ de diámetro, 2-4 areolas en $10 \mu \mathrm{m}$.

Metafiton en cursos de agua circulante salobre.

Loc.: 7 (VII/82).- 3 (IV/85).- 4 (IV/85).

\section{OR D E N P E N N A L E S SUBORDEN ARAPHIDINEAE \\ Familia DIATOMACEAE (FRAGILARIACEAE).}

Diatoma elongatum (Lyngbye) Agardh

Dimensiones: $20-29$ x $5 \mu \mathrm{m}$. Costillas: $3-6$ cada $10 \mu \mathrm{m}$ y $18-20$ estrías en $10 \mu \mathrm{m}$.

Epífita sobre plantas sumergidas en aguas alcalinas dulces o salobres, incluso polucionadas; no coincide con la ecología dada por Margalef (1954a) (Tabla III). 
Loc.: 3 (IV/84).- 9 (VII/83).- 10 (VIII/83).- 14 (VIII/85).- 19 (VII/83).-22 (IX/84).- 23 (VI/83).- 26 (IX/85).- 27 (IX/85).- 29 (III/84, XII/84).- 33 (VI/82).- 40 (IX/85).- 48 (VIII/85).- 55 (VIII/85).- 56 (VIII/85).- 57 (II/85).- 58 (VII/83).- 59 (VII/83).- 60 (XII/82).61 (VII/83.- 62 (VII/83).- 63 (VII/83).- 64 (VII/83).- 65 (I/82).- 66 (IX/84).- 67 (IX/84).68 (IX/84).- 69 (V/82).

Diatoma hiemale (Lyngbye) Heiberg var. mesodon (Ehrenberg) Grunow Dimensiones: $15-22 \times 6-9 \mu \mathrm{m}$. Tres costillas cada $10 \mu \mathrm{m}$.

Forma masas parduzcas sobre briófitos y otras plantas acuáticas en aguas muy puras de fuentes o en la cabecera de los ríos (Tabla III).

Loc.: 56 (VIII/85).- 62 (VII/83).- 20 (VIII/85).- 1 (VII/83).- 70 (VII/83).- 68 (IX/84).

Diatoma vulgare Bory

Células de $24-57$ x 5-8 $\mu \mathrm{m}$ con 7-8 costillas y $16-20$ estrías cada $10 \mu \mathrm{m}$.

Sobre plantas acuáticas en cursos de agua pura (Tabla III).

Loc.: 18 (II/85).- 56 (VII/85).- 57 (II/85).- 66 (IX/84).- 71 (VII/83).- 72 (II/85).

Meridion circulare (Greville) Agardh

Dimensiones: $40-50 \times 8-9 \mu \mathrm{m}$. Con 4 costillas y 15 estrías cada $10 \mu \mathrm{m}$.

Planctónico o epífito sobre otras algas en surgencias de aguas muy puras (Tabla III).

Loc.: 56 (VIII/85).- 62 (VII/83).- 73 (VII/83).

Licmophora gracilis (Ehrenberg) Grunow var. anglica (Kützing) Peragallo

Valvas de $45-50 \times 8-10 \mu \mathrm{m}$, con 24-28 estrías cada $10 \mu \mathrm{m}$.

Forma arbúsculos unidos por pedúnculos mucilaginosos sobre otras algas en la desembocadura del río (Tabla III).

Loc.; 74 (VIII/83).

Fragilaria capucina Desmazières

Valvas de 70-84 $\mu \mathrm{m}$, con 14-15 estrías cada $10 \mu \mathrm{m}$.

Planctónica o epífita sobre plantas acuáticas en aguas alcalinas dulces (Tabla III).

Loc.: 26 (III/84).- 62 (VII/83).- 65 (I/82).

Fragilaria construens (Ehrenberg) Grunow var. venter (Ehrenberg) Grunow

Valvas de $6 \times 5 \mu \mathrm{m}$, con 10 estrías cada $10 \mu \mathrm{m}$.

Aguas circulantes puras, sobre briófitos sumergidos (Tabla III).

Loc.: 9 (IX/85).- 26 (IX/85).- 56 (VII/85).

Synedra acus Kützing (=Fragilaria ulna (Nitzsch.) Lange Bertalot)

Valvas de $50-200 \times 6-10 \mu \mathrm{m}$, con $12-14$ estrías cada $10 \mu \mathrm{m}$.

Lange-Bertalot (1980) sinonimiza los géneros Fragilaria y Synedra.

Bentónicas sobre piedras o plantas sumergidas en aguas circulantes, dulces, impolutas. Penetra en agua salobre.

Loc.: 3 (IV/84, IX/84, IV/85).- 4 (IV/84).- 5 (IV/84, IV/85).- 6 (IV/84).- 7 (VII/83, IX/84).- 8 (IX/84).- 16 (VIII/85).- 22 (IV/84).- 26 (IX/84).- 27 (III/84).- 28 (III/84).- 29 (III/84, XII/84).- 30 (V/81).- 31 (VI/81).- 32 (V/82).- 40 (III/84, IX/85).- 41 (II/85).- 43 (I/83).- 51 (VII/83).- 53 (IV/84).- 57 (II/85).- 58 (VII/83).- 59 (VII/83).- 62 (VII/83).- 65 (III/81).- 68 (IX/84).- 75 (VI/83).- 76 (VI/83).- 77 (VII/83).- 78 (III/84).

var. radians (Kützing) Grunow

De menores dimensiones: $30 \times 2-3 \mu \mathrm{m}$. Convive con la especie.

Synedra pulchella Kützing (Fragilaria p. (Ralfs ex Kütz.) Lange Bertalot)

De 60-80 x 4-6 $\mu \mathrm{m}, 12-14$ estrías en $100 \mu \mathrm{m}$.

Epífita sobre algas filamentosas en cursos de agua salobre, En la cuenca no se comporta como eurioica como Margalef (1954a) sugiere.

Loc.: 7 (VII/82).- 3 (IV/85).- 4 (IV/85).- 6 (IV/84).- 8 (IV/84).- 51 (VII/83).- 64 (VII/83). 
Synedra tabulata Agardh (=Fragilaria t. (Ag.) Lange Berlatot) var. fasciculata (Kützing) Grunow

Valvas de $50-100 \times 3-7 \mu \mathrm{m}$ con 10-12 estrías cada $10 \mu \mathrm{m}$.

Forma haces alrededor de las algas filamentosas. Eurihalina. Resistente a la polución (Tabla'III).

Loc.: 2 (VIII/83).- 3 (IV/84).- 4 (IV/84).- 5 (VII/83, IX/84).- 6 (IV/84).- 7 (IX/84).- 14 (VIII/85).- 22 (IX/84).- 33 (VI/81).- 37 (VII/83).- 61 (VII/83).- 79 (VII/83).

Synedra ulna (Nitzsche) Ehrenberg (=Fragilaria u. (Nitzsch.) Lange Bertalot)

Valvas de hasta $500 \mu \mathrm{m}$ de longitud y $6-8 \mu \mathrm{m}$ de anchura, con 79 estrías cada $10 \mu \mathrm{m}$.

Sobre plantas sumergidas en agua circulante. Soporta la presencia de sal y la polución (Tabla III).

Loc.: 3 (IV/84).- 4 (IV/84).- 5 (VII/83).- 5 (VII/83).- 10 (VIII/83).- 14 (VIII/85).- 18 (VII/83).- 22 (XI/84, II/85, IV/85).- 25 (II/82).- 26 (IX/85).- 27 (III/84, IX/85).- 28 (III/84, XII/84).- 29 (III/84, XI/84). 30 (V/81).- 32 (V/82).- 33 (VI/81).- 36 (IV/84).- 40 (IV/84).56 (VIII/85).- 57 (II/85).- 59 (VII/83).- 65 (VI/81).- 66 (IX/84).- 68 (IV/84).- 69 (V/81).- 75 (VI/83).- 77 (VIII/83).- 78 (III/84).- 80 (IX/83).- 81 (VIII/85).- 82 (IX/83).- 83 (IX/83).- 84 (VII/83).- 85 (VII/83).- 86 (XI/84).- 88 (VII/83).- 89 (IX/84).

Las variedades danica (Kützing) Grunow y oxyrhynchus (Kützing) Van Heurck conviven muchas veces con la especie.

Synedra vaucheriae Kützing (= Fragilaria capucina v. vaucheriae (Kütz.) Lange-Bertalot).

Valvas de $12 \times 5 \mu \mathrm{m}$, con 12 estrías cada $10 \mu \mathrm{m}$.

Sobre plantas sumergidas en aguas circulantes puras (Tabla III).

Loc.: $56(\mathrm{VIII} / 85)$.

\section{SUBORDEN RAPHIDIOIDINEAE \\ Familia EUNOTIACEAE}

Eunotia arcus Ehrenberg

Dimensiones: $38-49$ × 5-9 $\mu \mathrm{m}$. Con 9-12 estrías en $10 \mu \mathrm{m}$.

Sobre plantas acuáticas en aguas circulantes calcáreas puras (Tabla III).

Loc.: 20 (VIII/85).- 22 (XI/84, II/85, VIII/85).- 23 (VI/83).- 26 (VII/84, IX/85).- 27 (IV/83).- 28 (III/84).- 29 (III/84).- 30 (V/81).- 36 (IV/84).- 40 (VIII/83).- 41 (II/85).- 55 (VIII/85).- 67 (VII/85).- 68 (IX/84).- 85 (VII/83).- 88 (VII/83).- 90 (II/85).

\section{SUBORDEN MONORAPHIDINEAE Familia ACHNANTACEAE}

Cocconeis pediculus Ehrenberg

Dimensiones: $15-40 \times 10-25 \mu \mathrm{m}$. Con $15-17$ estrías cada $10 \mu \mathrm{m}$.

Epífito sobre plantas acuáticas en aguas circulantes, calcáreas dulces o salobres, como ya indicaba Margalef (1954a). Soporta la polución (Tabla III).

Loc.: 3 (IV/84, IX/84, XI/84, IV/85).- 4 (VIII/83).- 5 (IV/84, IV/85).- 6 (IV/84).- 7 (VII/83, IX/84).- 8 (IX/84).- 18 (VII/83).- 19 (VII/83).- 22 (II/85, IV/85).- 25 (II/82).- 26 (III/84, IX/85).- 28 (XII/84).- 30 (V/82).- 31 (I/82).- 33 (XI/81).- 36 (IV/84).- 40 (IX/85).41 (II/85).- 46 (IX/83).- 57 (II/85).- 59 (VII/83).- 63 (VII/83).- 65 (VI/82).- 66 (IV/84).- 68 (VII/83, IV/84).- 71 (VII/83).- 75 (VI/83).- 81 (VIII/85).- 89 (VII/83).- 91 (VII/83).- 92 (VII/83).- 93 (VII/83).- 94 (VI/83).- 95 (VII/83).- 96 (VII/83).

Cocconeis placentula Ehrenberg

Dimensiones: $20-40 \times 10-20 \mu \mathrm{m}$. Con $15-20$ estrías cada $10 \mu \mathrm{m}$.

Epífito sobre macrófitos en aguas circulantes o paredes rezumantes en aguas calcáreas puras. (Tabla III). En la cuenca no se ha recolectado en aguas salobres.

Loc.: 14 (VIII/84).- 17 (VII/83).- 22 (IX/84, XI/84, II/85, IV/85).- 25 (II/82).- 26 (IX/85).- 27 (III/84).- 28 (III/84).- 29 (III/84).- 20 (V/81).- 33 (VI/82).- 40 (III/84 ).- 41 (II/85).- 68 (IV/84).- 97 (VI/82). 
Achnanthes brevipes Agardh

Dimensiones: $35-65 \times 12-20 \mu \mathrm{m}$, con 8-9 estrías cada $10 \mu \mathrm{m}$.

Tanto el tipo como la v. intermedia (Kütz.) Cleve y v. parvula (Kütz.) Cleve viven epífitas sobre plantas acuáticas en cursos de agua salobre (Tabla III).

Loc.: 2 (VII/83).- 3 (IV/83).- 4 (VI/83).- 5 (VI/83).- 6 (VI/83).- 7 (VII/83).- 8 (IX/84).14 (VIII/85).- 53 (VI/83).- 61 (VIII/85).- 79 (VII/83).- 98 (IX/85).

Achnanthes flexella (Kützing) Braun

Dimensiones: 23 x $12 \mu \mathrm{m}$. Estrías 24-30 en $10 \mu \mathrm{m}$..

Sobre plantas y otros substratos sumergidos en charcas o cursos de agua calcárea no contaminada (Tabla III).

Loc.: 23 (VI/83).- 24 (VII/83).- 26 (III/84, IV/84, IX/85).- 27 (IV/83).- 28 (III/84).- 29 (III/84).- 36 (IV/84).- 40 (III/84, IX/85).- 88. (VII/83).

Achnanthes microcephala Kützing

Dimensiones: 20 x 3-4 $\mu \mathrm{m}$. Estrías: $30-35$ en $10 \mu \mathrm{m}$.

Epífita en cursos de agua dulce alcalina.

Loc.: 41 (III/85).- 62 (VII/83).

Achnanthes minutissima Kützing

Se diferencia de la anterior por la ausencia de área central.

Sobre algas filamentosas en cursos de agua alcalina dulce, a veces bastante mineralizada (Tabla III).

Loc.: 1 (VII/83).- 20 (VII/83).- 22 (IX/84, II/85).- 25 (II/82).- 26 (IX/85).- 27 (III/84, IX/85).- 28 (IX/85).- 29 (III/84).- 30 (V/81).- 31 (I/82).- 32 (V/82).- 36 (IV/84).- 40 (III/84).- 55 (VIII/85).- 65 (I/82).- 66 (IX/84).- 68 (X/84).- 87 (VIII/85).- 88 (VII/83).- 99 (VII/83).

Rhoicosphenia curvata (Kützing) Grunow

Dimensiones: $22-24$ x 5-8 $\mu \mathrm{m}$ con 13-15 estrías en cada $10 \mu \mathrm{m}$.

Fija a plantas y objetos sumergidos en cursos de agua dulce o más frecuentemente salobre (Tabla III).

Loc.: 3 (IV/84, IX/84).- 4 (IV/84).- 5 (IV/84).- 14 (VIII/85).- 22 (XI/84, II/85, IX/85).26 (III/84).- 29 (XII/84).- 31 (V/81).- 41 (II/85).- 53 (IV/84).

\section{SUBORDEN BIRAPHIDINEAE Familia NAVICULACEAE}

\section{Mastogloia aquilegiae Grunow}

Dimensiones: $67 \times 22 \mu \mathrm{m}$ con 16 estrías cada $10 \mu \mathrm{m}$.

Sobre plantas u otros substratos sumergidos en aguas salobres circulantes (Tabla IV). Hustedt (1959) la considera marina, Tomas (1981) la recolecta en numerosas charcas y lagunas litorales mediterráneas sobre todo en el sur de la península.

Loc.: 3 (IV/83).- 4 (VII/83).- 5 (IV/85).

Mastogloia baltica Grunow

Dimensiones: $42 \times 17 \mu \mathrm{m}$ con 20 estrías cada $10 \mu \mathrm{m}$.

Sobre plantas acuáticas de aguas salobres circulantes. Más rara que la anterior convive con ella (Tabla IV). Es frecuente en charcas del litoral mediterráneo español (Tomas, 1981).

Loc.: 3 (XI/84).

Mastogloia braunii Grunow .

Dimensiones: $55 \times 20 \mu \mathrm{m}$ con 17 estrías cada $10 \mu \mathrm{m}$.

Epífita sobre algas filamentosas de aguas salobres circulantes (Tabla IV). Está ampliamente distribuida por todo el litoral español mediterráneo (Tomas, 1981).

Loc.: 3 (IV/84).- 4 (VII/85).- 5 (IV/84, IV/85).- 6 (IV/84).- 7 (VII/83).- 22 (IX/84, IV/85, VIII/85).- 60 (VIII/85).- 64 (VII/83).- 86 (XI/84). 
Mastogloia elliptica Agardh

De $20-30 \times 10-15 \mu \mathrm{m}$, con 16 estrías en $10 \mu \mathrm{m}$.

Epífita sobre clorofíceas filamentosas en cursos salobres. Para Germain (1981) se comporta como eurihalina.

Loc.: 3 (IV/85).- 6 (IV/84).

Mastogloia pumila (Grunow) Cleve

De 20-25 x 5-8 $\mu \mathrm{m}$, con 25 estrías en $10 \mu \mathrm{m}$.

Epífita sobre clorofíceas filamentosas en cursos de agua salobre, junto a otras especies congéneres.

Loc.: 3 (IV/85).- 6 (IV/84).- 8 (IV/84).

Mastogloia recta Hustedt

Dimensiones.: $45 \times 12 \mu \mathrm{m}$, con 14 estrías cada $10 \mu \mathrm{m}$.

Sobre objetos sumergidos en aguas dulces circulantes no polucionadas (Tabla IV). Tomas (1981) la considera más típica de aguas medianamente mineralizadas,

Loc.: 26 (III/84).- 100 (XI/81).

Mastogloia smithii Thwaites var. lacustris Grunow

Dimensiones: $45 \times 12 \mu \mathrm{m}$ con 14-16 estrías cada $10 \mu \mathrm{m}$.

Sobre plantas acuáticas u otros objetos sumergidos en cursos de agua dulce o salobre no contaminados (Tabla IV).

Loc.: 3 (IV/84, IX/84).- 5 (IV/84).- 22 (III/85, IV/85).- 24 (VIII/83).- 26 (IV/83).- 27 (III/84, IX/85).- 40 (IX/85).- 41 (II/85).- 101 (IV/82).

Pleurosigma elongatum W. Smith

Dimensiones: $180-195 \times 20 \mu \mathrm{m}$ con $16-20$ estrías cada $10 \mu \mathrm{m}$.

Herpon de cursos de agua salobre. Soporta la polución (Tabla IV).

Loc.: 3 (IX/84).- 4 (IV/84, IV/85).- 5 (IV/84, IV/85).- 6 (IV/84).- 7 (VII/83).- 8 (IX/84).- 53 (IV/84).- 60 (VI/83).- 86 (XI/84).- 102 (III/81).

Gyrosigma acuminatum (Kützing) Rabenhorst

Dimensiones: $124 \times 18 \mu \mathrm{m}$. Con $18-20$ estrías cada $10 \mu \mathrm{m}$.

Sobre plantas u otros objetos sumergidos en aguas dulces o salobres no polucionadas. Eurioica (Tabla IV).

Loc.: 2 (VII/83).- 3 (IX/84).- 4 (IV/84).- 26 (IX/85).- 27 (IV/83).- 28 (III/84, IX/85).30 (V/81).- 31 (I/82).- 33 (IV/82).- 34 (XI/81).- 60 (VII/83).- 66 (IX/84).- 75 (VI/83).- 103 (VII/85).

Gyrosigma scalproides (Rabenhorst) Cleve

Valvas de 40-50 $\mu \mathrm{m}$ de longitud, con 22-24 estrías transversales y 28-30 estrías longitudinales en $10 \mu \mathrm{m}$.

Sobre plantas acuáticas en aguas salobres y más raramente en aguas dulces carentes de polución.

Loc.: 3 (VII/83).- 4 (IV/84).- 5 (IV/84).- 6 (IV/84).- 7 (IX/84).- 26 (III/84, IV/85).- 35 (IX/84).- 60 (XII/82).- 64 (VII/83).- 75 (VII/83).

Amphiprora alata Kützing $10 \mu \mathrm{m}$.

Valvas de 35-50 $\mu \mathrm{m}$ de longitud. Cíngulo de $14 \mu \mathrm{m}$ de anchura. Con 17-19 estrías cada

Sobre plantas u otros objetos sumergidos. Eurihalina. Soporta la polución orgánica (Tabla IV).

Loc.; 4 (XI/84).- 38 (VIII/85).- 102 (III/81).- 14 (XII/82).

Amphipleura pellucida Kützing

Dimensiones hasta $140 \times 7-9 \mu \mathrm{m}$. Hasta 40 estrías cada $10 \mu \mathrm{m}$.

Entre las plantas ribereñas en aguas dulces no polucionadas (Tabla IV).

Loc.: 20 (IX/84).- 22 (XI/84, II/85).- 26 (III/84, IV/84, IX/85).- 27 (III/84).- 28 (III/84).- 29 (III/84, XII/84).- 31 (I/82).- 33 (III/81, IV/82).- 35 (IX/84).- 41 (III/85).- 59 (VIL/83).- 66 (IX/84).- 68 (IV/84). 
Diploneis coffeaiformis (A. Schmidt) Cleve

Dimensiones: $25 \times 15 \mu \mathrm{m}$ con 11 estrías cada $10 \mu \mathrm{m}$.

Sobre algas filamentosas en cursos de agua salobre (Tabla IV).

Loc.: 3 (IV/84, IX/84).

Diploneis didyma (Ehrenberg) Cleve

Dimensiones: $50 \times 20 \mu \mathrm{m}$, con 14 estrías cada $10 \mu \mathrm{m}$.

Epífita sobre clorofíceas filamentosas en cursos de agua salobre (Tabla IV).

Loc.: 3 (IV/84).- 4 (VII/83).- 5 (VI/83).- 6 (IV/84).

Diploneis elliptica Kützing

Dimensiones: $25-60 \times 12-25 \mu \mathrm{m}, 8-10$ estrías cada $10 \mu \mathrm{m}$.

Aerófila o entre las plantas sumergidas en agua dulce (Tabla IV).

Loc.: 22 (II/85, IV/85).- 26 (III/84, IX/85).- 27 (IV/83, III/84, IX/85).- 28 (III/84, IX/85).- 29 (III/84, XII/84, XII/84).- 31 (VI/82).- 34 (XI/81).- 40 (VII/83).- 58 (VIII/83).63 (VII/83).- 65 (VI/82).- 69 (IV/81).- 78 (III/84).- 85 (VII/83).- 88 (VIII/85).- 97 (VI/82).

\section{Diploneis ovalis Kützing}

Dimensiones; $30-90 \times 10-41 \mu \mathrm{m}, 11-14$ estrías cada $10 \mu \mathrm{m}$.

En condiciones similares a la especie anterior y conviviendo con ella en muchas ocasiones. Puede penetrar sin embargo en agua salobre, como ya había indicado Margalef (1954a). (Tabla IV).

Loc.: 3 (IV/84).- 4 (IV/84).- 5 (IV/84).- 6 (IV/84).- 7 (IV/84).- 22 (IV/85, VII/85).- 24

(VII/83).- 26 (IV/85, IX/85).- 27 (IV/83.- 28 (III/84).- 29 (III/84, XII/84).- 31 (I/82).- 41

(II/85).- 53 (IV/84).- 75 (VI/83).

var. oblongella (Näegeli) Cleve

De menor tamaño: $20-25 \times 10 \mu \mathrm{m}$ con $18-20$ estrías cada $10 \mu \mathrm{m}$.

Sólo en agua salobre (Tabla IV).

Loc.: 3 (IV/84).- 4 (IV/84).- 5 (IV/84).- 6 (IV/84).- 6 (IV/84).- 7 (IV/85).- 53 (IV/84).

Diploneis puella (Schumann) Cleve

Dimensiones: 17 x $8 \mu \mathrm{m}, 16-17$ estrías cada $10 \mu \mathrm{m}$.

Entre plantas ribereñas en aguas dulces circulantes no polucionadas (Tabla IV), Germain (1981) la considera aer6fila.

Loc.: 26 (V/85).- 28 (IX/85).- 40 (III/84, IX/85).

Diploneis smithii (Brébisson) Cleve

Valvas de hasta $90 \times 40 \mu \mathrm{m}, 8$ estrías en $10 \mu \mathrm{m}$.

Epífita sobre algas filamentosas en cursos de agua salobre. (Tabla IV). Germain (1981) la considera eurihalina.

Loc.: 3 (IV/84).- 4 (IV/84, IV/85).- 6 (IV/84).

Neidium dubium (Ehrenberg) Cleve

Dimensiones: $40 \times 13 \mu \mathrm{m}, 20-22$ estrías en $10 \mu \mathrm{m}$.

En riberas de cursos de agua dulce alcalina no polucionada entre macrófitos (Tabla IV).

Loc.: 26 (IX/85).

Stauroneis anceps Ehrenberg

Dimensiones: $40-50 \times 15 \mu \mathrm{m}, 20-25$ estrías en $10 \mu \mathrm{m}$.

Especie ribereña en cursos de agua alcalina dulce no polucionada. Herpon (Tabla IV).

Para Margalef (1954a) se trata de una especie calcífuga, por el contrario.

Loc.: 26 (III/84).- 94 (VIII/85).- 95 (VII/85).

Stauroneis phoenicenteron Ehrenberg

Dimensiones: 125 x $30 \mu \mathrm{m}, 16-17$ estrías en $10 \mu \mathrm{m}$.

Entre los macrófitos de cursos de agua dulce alcalina no polucionada (Tabla IV). No se comporta como calcífuga, como sugiere Margalef (1954a).

Loc.: 26 (III/84).- 27 (IV/83).- 40 (IX/85). 
Stauroneis salina W. Smith

Dimensiones: $70-75 \times 8-10 \mu \mathrm{m}, 20-25$ estrías cada $10 \mu \mathrm{m}$.

Entre plantas acuáticas en ramblas salobres (Tabla IV).

Loc.: 4 (XI/84).- 60 (XII/82).- 64 (VII/83).

Stauroneis spicula Hickie

De $50-80 \times 4-10 \mu \mathrm{m}$, con 25 estrías en $10 \mu \mathrm{m}$.

En relación con algas filamentosas en cursos de agua salobre.

Loc.: 7 (VII/82).

Anomoeoneis sphaerophora (Ehrenberg) Pfitzer

Dimensiones: $50-55 \times 18-20 \mu \mathrm{m}, 18$ estrías cada $10 \mu \mathrm{m}$.

Entre macrófitos ribereños en agua dulce, estancada o circulante (Tabla IV). Para Germain (1981) puede considerarse eurihalina.

Loc.: 9 (IX/85).- 63 (VII/83).

Navicula bacillum Ehrenberg

Dimensiones: $22 \times 8 \mu \mathrm{m}, 16$ estrías en $10 \mu \mathrm{m}$.

Herpon de aguas alcalinas, dulces, no contaminadas (Tabla V).

Loc.: 22 (II/85).

Navicula contenta Grunow f. biceps Arnot

Dimensiones: $10-12 \times 2-3 \mu \mathrm{m}, 30-35$ estrías en $10 \mu \mathrm{m}$.

Sobre plantas acuáticas en riberas de cursos de agua alcalina dulce (Tabla V).

Loc.: 40 (VIII/83).

Navicula cryptocephala Kützing

De $25-30 \times 5-7 \mu \mathrm{m}$, con 16-17 estrías cada $10 \mu \mathrm{m}$.

Relacionada con algas filamentosas de cursos de agua salobre aunque según Germain (1981) se trata de una especie eurihalina.

Loc.: 7 (VII/82). 8 (IV/84).

Navicula cuspidata Kutzing v. ambigua (Ehrenberg) Cleve

Dimensiones: $80-82 \times 20-24 \mu \mathrm{m}, 18$ estrías transversales y 20-25 longitudinales en 10 $\mu \mathrm{m}$.

Herpon de riberas de cursos de agua dulce. Soporta la presencia de sal y la polución (Tabla V).

Loc.; 26 (IV/84).- 61 (VIII/83).

Navicula gracilis Ehrenberg

De $30-50 \times 7-8 \mu \mathrm{m}$, con 10 estrías en $10 \mu \mathrm{m}$.

En relación con algas filamentosas en cursos de agua salobre.

Loc.: 7 (VII/82).- 4 (IV/85).- 43 (VI/83).

Navicula lanceolata (Agardh) Ehrenberg

Dimensiones: $35-40 \times 10-12 \mu \mathrm{m}, 9-10$ estrías en $10 \mu \mathrm{m}$.

Dentro de tubos mucosos sobre rocas en cursos de agua alcalina dulce, no contaminada (Tabla V).

Loc.: 26 (III/84).

Navicula oblonga Kützing

Dimensiones: $175 \times 20 \mu \mathrm{m}, 6-7$ estrías en $10 \mu \mathrm{m}$.

Charcas permanentes de agua alcalina dulce no polucionada (Tabla V). Margalef (1954a)

la encuentra en aguas bastante mineralizadas.

Loc.: 26 (III/84).

Navicula radiosa Kützing

Dimensiones: $70-90 \times 13-15 \mu \mathrm{m}, 9-11$ estrías en $10 \mu \mathrm{m}$.

Muy común en charcas y riberas de arroyos de agua alcalina dulce. Bentónica. Penetra en agua salobre. Sensible a la polución. 
Loc.: 20 (VIII/85).- 27 (IV/85).- 31 (III/82).- 33 (I/82).- 34 (II/82).- 40 (VIII/85).- 64 (VII/85).- 67 (IX/84).- 68 (IX/84).- 75 (VI/83).- 85 (VII/83).- 87 (VIII/85).- 94 (VIII/85).104 (VIII/85).

Navicula rhynchocephala Kützing

Dimensiones: $22-48 \times 12-17 \mu \mathrm{m}, 14$ estrías cada $10 \mu \mathrm{m}$.

Sobre diversos substratos sumergidos en riberas de cursos de agua alcalina dulce no contaminada (Tabla V).

Loc.: 26 (IX/85).- 65 (III/82).- 88 (VII/83).

Navicula schroeteri Meister

Dimensiones; $40 \times 7-8 \mu \mathrm{m}, 12-13$ estrías cada $10 \mu \mathrm{m}$.

Charcas permanentes de agua alcalina dulce, de elevada mineralización. Herpon (Tabla

V). Germain (1981), también la recolecta en agua salobre.

Loc.: 22 (II/85).

Caloneis silicula (Ehrenberg) Cleve

Dimensiones: $35 \times 8 \mu \mathrm{m}$. Con $18-20$ estrías cada $10 \mu \mathrm{m}$.

Herpon de charcas de agua alcalina dulce (Tabla VI).

Loc.: 26 (V/85).

Caloneis ventricosa (Ehrenberg) Meister

Dimensiones: $83 \times 15 \mu \mathrm{m}, 19-21$ estrías cada $10 \mu \mathrm{m}$.

Herpon de charcas de agua dulce alcalina (Tabla VI).

Loc.: 63 (VII/83).- 40 (II/84, IX/85).- 26 (II//84, IX/85).- 27 (IX/85).

Pinnularia brebissonii (Kützing) Rabenhorst

Dimensiones: $50 \times 10-12 \mu \mathrm{m}$, con 10-12 estrías cada $10 \mu \mathrm{m}$.

Sobre objetos sumergidos de arroyos con agua alcalina dulce, no polucionada, aunque según Germain (1981) soporta bien la polución (Tabla VI).

Loc.: 26 (III/84).- 78 (III/84).

Pinnularia mesolepta (Ehrenberg) W. Smith

Dimensiones: $54 \times 10 \mu \mathrm{m}$, con 10-11 estrías cada $10 \mu \mathrm{m}$. VI).

Herpon en remansos de arroyos de agua aicalina dulce. Resistente a la polución (Tabla

Loc.: 14 (VIII/85).- 66 (VII/85).- 26 (III/84).- 41 (II/85).- 31 (I/82).

Pinnularia viridis (Nitzsche) Ehrenberg

Dimensiones: $190 \times 34 \mu \mathrm{m}$ con 7 estrías cada $10 \mu \mathrm{m}$.

Herpon de charcas y cursos de agua alcalina dulce. Soporta bien la desecación como ya indicaba Germain (1981). (Tabla VI).

Loc.: 63 (VII/83).- 52 (VI/82).- 26 (III/84, IV/84, IX/84).- 29 (III/84).- 27 (IX/85).- 22 (IX/84).- 41 (II/85).- 35 (IV/84).

Cymbella affinis Kützing

Dimensiones: $30-40 \times 10 \mu \mathrm{m}$ con 8 estrías cada $10 \mu \mathrm{m}$.

Forma masas pulviniformes sobre las rocas de cursos de agua alcalina salobre (Tabla VI). Coincide con la ecología dada por Tomas (1979).

Loc.: 51 (VII/83).

Cymbella caespitosa (Kützing) Brunnthaler

Dimensiones: $35 \times 13 \mu \mathrm{m}$, con 10 estrías cada $10 \mu \mathrm{m}$.

En el interior de tubos mucosos sobre plantas sumergidas o rocas en arroyos de agua alcalina (Tabla VI).

Loc.: 105 (VII/83).- 94 (VII/83).- 95 (VII/83).- 26 (III/84, XII/84).

Cymbella lanceolata (Ehrenberg) Van Heurck

Dimensiones: $79-105 \times 15-23 \mu \mathrm{m}$ con 6-10 estrías cada $10 \mu \mathrm{m}$. 
Fija mediante pedúnculos gelatinosos largos y ramificados a las plantas sumergidas, en arroyos de agua alcalina dulce (Tabla VI). Coincide plenamente con la ecología dada por Tomas (1979).

Loc.: 57 (VII/83).- 106 (VI/83).- 25 (II/82).- 40 (III/84, IX/85).- 27 (III/84).- 26 (V/83, IX/84, XII/84).- 28 (III/84, IX/85).- 29 (III/84, XI//84).- 42 (II/85).- 35 (IV/84).

Cymbeila leptoceros (Ehrenberg) Grunow

Dimensiones: $46-47 \times 18,8-10$ estrías cada $10 \mu \mathrm{m}$ en la parte ventral y 10 estrías en 10 $\mu \mathrm{m}$ en la dorsal.

Riberas de cursos de agua alcalina dulce (Tabla VI).

Loc.: 52 (III/81).

Cymbella parva (W. Smith) Cleve

Dimensiones: $70 \times 15 \mu \mathrm{m}$. Cada $10 \mu \mathrm{m}$ posee 9 estrías en la parte ventral y $8-9$ en la dorsal.

Cursos de agua alcalina dulce.

Loc.: 27 (III/84).- 32 (V/82).

Cymbella prostrata (Berkeley) Cleve

Dimensiones: $75 \times 25 \mu \mathrm{m}$. Cada $10 \mu \mathrm{m}$ presenta 7-10 estrías en la parte ventral y 8-9 en la dorsal.

En el interior de tubos mucosos, sobre plantas u objetos sumergidos en agua alcalina dulce (Tabla VI). Según Hustedt (1930) puede soportar la presencia de sal.

Loc.: 106 (VI/83).- 40 (III/84).

Cymbella pusilla Grunow

De 30-50 x $5 \mu \mathrm{m}$ con 10-12 estrías en $10 \mu \mathrm{m}$.

Relacionada con algas filamentosas en cursos de agua salobre. Gemain (1981) la recolecta siempre cerca del mar.

Loc.: 7 (VII/82).

Cymbella tumida (Brébisson) Van Heurck

Dimensiones: $40 \times 12 \mu \mathrm{m}, 8-10$ estrías en $10 \mu \mathrm{m}$.

Sobre largos pedúnculos en piedras y plantas sumergidas formando masas algodonosas pardas en agua alcalina dulce, de elevada mineralización en ocasiones (Tabla VI).

Loc.: 87 (VIII/85).- 108 (VIII/85).- 57 (II/85).- 58 (VIII/83).- 59 (VII/83).- 9 (VIII/83).23 (VI/83).- 104 (VIII/85).- 24 (VIII/83).- 95 (VII/83).- 109 (VII/83).- 62 (VII/83). 110 (VII/83).- 75 (VI/83).- 76 (VI/83).- 111 (VIII/85).- 64 (VII/83).- 25 (II/82).- 40 (VIII/83).26 (III/84, IV/84, IX/84, IV/85, IX/85).- 33 (VI/82).- 65 (VI/82).- 28 (III/84).- 27 (III/84, IX/85). 29 (III/84, XI/84).- 78 (III/84).- 41 (II/85).- 22 (IX/84, XI/84, IV/85).- 30 (V/81).31 (IV/81, I/82).- 69 (V/81).- 66 (IX/84).

\section{Cymbella ventricosa Kützing}

Dimensiones: $20 \times 7-8 \mu \mathrm{m}$ con 10-12 estrías cada $10 \mu \mathrm{m}$.

Sobre plantas acuáticas en agua alcalina dulce (Tabla VI).

Loc.; 56 (VII/85).- 90 (II/85).- 116 (VI/83).- 113 (VII/83).- 26 (IX/85).

\section{Amphora acutiuscula Kützing}

De $30-50 \mu \mathrm{m}$ de longitud, con $15-16$ estrías cada $10 \mu \mathrm{m}$.

Relacionada con algas filamentosas en cursos de agua salobre.

Loc.: 3 (IV/85).- 7 (VII/82).- 8 (IV/84).

Amphora coffeaeformis Agardh

De $20-50 \mu \mathrm{m}$ de longitud, con 20 estrías cada $10 \mu \mathrm{m}$.

En cursos de agua salobre en relación con algas filamentosas. Coincide con los datos de Margalef (1954b).

Loc: 3 (IV/85).- 7 (VII/82).- 4 (IV/85).- 6 (IV/84).- 43 (VI/83).- 51 (VII/83).

De $40-60 \mu \mathrm{m}$ de longitud con $10-11$ estrías cada $10 \mu \mathrm{m}$. 
Relacionada con algas filamentosas en cursos de agua salobre. Germain (1981) sólo la encuentra en la costa. Coincide con la descripción y ecología de Schoeman \& Archibald (1986)

Loc.: 3 (IV/85).

Amphora holsatica Hustedt

Dimensiones: $45 \times 18 \mu \mathrm{m}$, con $14-15$ estrías cada $10 \mu \mathrm{m}$.

Bentos de cursos de agua alcalina salobre (Tabla VI).

Loc.: 60 (XII/82).- 51 (VII/83).- 3 (IV/84, IX/84, XI/84).- 4 (IV/84, IV/85).- 6 (IV/84).5 (IV/84).- 7 (VII/83, IX/84).- 8 (IX/84, IV/85).- 86 (XI/84).

Amphora lineolata Ehrenberg

Dimensiones: $43-60 \times 5-8 \mu \mathrm{m}$, con 10-12 estrías cada $10 \mu \mathrm{m}$.

Herpon de arroyos de agua salobre.

Loc.: 51 (VII/83).

Amphora normanii Rabenhorst

Dimensiones: $42 \times 7 \mu \mathrm{m}$ con $16-18$ estrías cada $10 \mu \mathrm{m}$.

Riberas de cursos de agua dulce alcalina. Herpon (Tabla VI). Parece resistir bien la desecación (Gemain, 1981).

Loc.: 28 (III/84).

Amphora ovalis Kützing

Dimensiones: $30-60 \times 15-20 \mu \mathrm{m}$ con $12-14$ estrías cada $10 \mu \mathrm{m}$.

Bentos de cursos de agua alcalina dulce o salobre (Tabla VI). Ya en Margalef (1954b) se comentaba su carácter eurioico.

Loc.; 115 (VII/83).- 64 (VII/83).

Amphora pediculus Kützing

De $15 \times 4 \mu \mathrm{m}$, con 17 estrías en $10 \mu \mathrm{m}$.

Epífita sobre clorofíceas filamentosas en arroyos salinos..

Loc.: 7 (VII/82).- 5 (IV/84).

Amphora proteus Gregory

De $100 \times 40 \mu \mathrm{m}$, con 6-8 estrías cada $10 \mu \mathrm{m}$.

En relación con clorofíceas filamentosas en cursos de agua salobre.

Loc.: 7 (VII/82).- 3 (IV/85).- 6 (IV/84).

Amphora veneta Kützing

De $30-50 \times 7-15 \mu \mathrm{m}$, con 20-25 estrías cada $10 \mu \mathrm{m}$.

Epífita sobre algas filamentosas en arroyos salinos. Para Gemain (1981) se trata de una especie eurihalina. (VII/83).

Loc.: 7 (VII/82).- 3 (IV/84).- 6 (IV/84).- 4 (IV/85).- 5 (IV/84).- 43 (VI/83).- 64

Gomphonema acuminatum Ehrenberg

Dimensiones: $58 \times 14 \mu \mathrm{m}$ con $10-12$ estrías cada $10 \mu \mathrm{m}$.

var. brebissonii (Kützing) Cleve

Ambas viven epífitas sobre plantas u otros objetos sumergidos en aguas alcalinas dulces (Tabla VI).

Loc.: 40 (IX/85).- 27 (IV/83, III/84).- 29 (III/84, XII/84).- 22 (IX/84).

Gomphonema constrictum Ehrenberg

Dimensiones: $50 \times 10 \mu \mathrm{m}$ con 9-12 estrías cada $10 \mu \mathrm{m}$.

var. capitata (Ehrenberg) Cleve

Ambas viven sobre plantas sumergidas u otros objetos en cursos de agua dulce alcalina (Tabla VI). Según Margalef (1954 b) la var. capitata prefiere aguas más eutróficas.

Loc.: 55 (VIII/85).- 57 (VI/83).- 88 (VII/83).- 84 (VII/83).- 62 (VII/83).- 18 (VI/83).111 (VIII/85).- 25 (II/82).- 28 (III\&84).- 33 (VI/82).- 1 (VII/83).- 26 (IV/83, VII/83, III/84, IV/84).- 27 (IV/83, III/84, IV/84).- 40 (III/84).- 29 (III/84, XII/84).- 22 (II/85).- 34 (XI/81).- 68 (IV/84). 


\section{Gomphonema intricatum Kützing}

De $50-70 \times 6-13 \mu \mathrm{m}$ con 8-10 estrías cada $10 \mu \mathrm{m}$.

Sobre objetos sumergidos en agua dulce alcalina estancada o circulante (Tabla VI).

Loc.: 91 (VII/83).- 88 (VII/83).- 62 (VII/83).- 63 (VII/83).- 40 (VIII/85).- 26 (IX/85).27 (IV/83, IX/85).- 28 (IX/85).- 29 (III/84, XII/84).- 22 (XI/84, II/85, IV/85).- 35 (IV/84, IX/85).- 115 (IX/84).

Gomphoneis olivacea (Lyngbye) Dawson. (Syn: Gomphonema olivaceum Lyngbye).

De $25 \times 8 \mu \mathrm{m}$ con 12 estrías cada $10 \mu \mathrm{m}$.

Forma masas pardas constituídas por pedúnculos mucosos ramificados que portan los frústulos. Cursos de agua alcalina dulce (Tabla VI). Coincide plenamente con las apreciaciones de Germain (1981).

Loc.: 90 (II/85).- 57 (II/85).- 41 (II/85).

Denticula elegans Kützing

De $20-22$ x $5 \mu \mathrm{m}$. Cada $10 \mu \mathrm{m}$ presenta 5 costillas transversales y 14-17 estrías.

Bentos de cursos de agua alcalina dulce o salobre (Tabla VI). Difiere en los datos ecológicos con Germain (1981) y Margalef (1954b) que sólo la citan de aguas dulces.

Loc.: 62 (VII/83).- 63 (VII/83).- 25 (II/82):- 40 (VIII/83).- 26 (III/84, IX/85).- 28 (IX/85).- 33 (VI/82).- 116 (VI/83).- 27 (IV/83).- 29 (XII/84).- 65 (I/82).- 31 (I/82). 30 (V/81).- 53 (IV/84).- 6 (IV/84).- 3 (IV/84).- 3 (IV/84, IX/84, XI/84).- 5 (IV/84).- 7 (IX/84).

Denticula tenuis Kützing var. crassula Näegeli

De $15 \times 7 \mu \mathrm{m}$, con 7-8 costillas y $25-30$ estrías cada $10 \mu \mathrm{m}$.

Epífita sobre plantas sumergidas en agua alcalina dulce (Tabla VI).

Loc.: 28 (IX/84).- 27 (III/84).- 29 (III/84).

\section{Epithemia argus Kützing}

De $110 \times 23 \mu \mathrm{m}$, con 2-3 costillas y 10-11 estrías en $10 \mu \mathrm{m}$.

Bentónica en cursos de agua dulce alcalina, a veces muy mineralizada o incluso salobres. Margalef (1954b) ya comentaba su preferencia por aguas muy mineralizadas. (Tabla VI).

Loc.: 23 (VI/83).- 24 (VIII/83).- 92 (VII/83).- 62 (VII/83).- 26 (III/84, IX/85).- 22 (IV/85, VIII/85).- 28 (IX/85).- 27 (III/84, IX/85).- 40 (VIII/83).- 29 (III/84).- 35 (IV/84). 68 (IV/84).- 3 (IV/84).

\section{Epithemia sorex Kützing}

De $35-40 \mu \mathrm{m}$, con 2-5 costillas y 10 estrías cada $10 \mu \mathrm{m}$.

Sobre plantas sumergidas en agua alcalina dulce (Tabla VI). Puede penetrar en aguas salobres (Margalef, 1954b).

Loc.: 24 (VII/83).

\section{Epithemia turgida (Ehrenberg) Kützing}

De $45-68 \times 13 \mu \mathrm{m}$, con 3-5 costillas y 10-12 estrías cada $10 \mu \mathrm{m}$.

Sobre plantas y otros objetos sumergidos en agua dulce alcalina (Tabla VI).

Loc.: 55 (VIIL/85).- 91 (VII/83).- 88 (VII/83).- 26 (IX/84).- 68 (IX/85).- 41 (IX/85).

Epithemia zebra (Ehrenberg) Kützing v. porcellus (Kützing) Grunow

De $30-50$ x 6-8 $\mu \mathrm{m}$, con 3-4 costillas y $8-10$ areolas cada $10 \mu \mathrm{m}$. (1981)

Epífita sobre algas filamentosas en arroyos alcalinos salobres, como ya indicaba Germain

Loc.: 3 (IV/85).

Rhopalodia gibba (Ehrenberg) O. Müller

De $90 \times 23 \mu \mathrm{m}$, con 8 costillas y $16-18$ estrías en $10 \mu \mathrm{m}$.

Entre algas filamentosas en cursos de agua alcalina dulce (Tabla VI).

Loc.: 33 (XI/81).- 22 (II/85, V/85, VIII/85).- 88 (VIII/85).- 40 (IX/85).- 27 (IV/83, III/84, IX/85).- 26 (IV/83, III/84, IX/84, V/85).- 28 (III/84, XII/84).- 78 (III/84).- 41 (II/85).- 100 (XI/81).- 35 (IX/84).- 3 (IV/84, XI/84). 
Rhopalodia gibberula (Ehrenberg) O. Müller

De $25-30$ x $8 \mu \mathrm{m}$, con $2-3$ costillas y $14-16$ estrías en $10 \mu \mathrm{m}$.

Bentónica en cursos de agua salobre o muy mineralizada (Tabla VI). Coincide con las observaciones de Margalef (1954b).

Loc.: 98 (IX/85).- 3 (IX/84, XI/84).- 26 (III/84, IX/84, V/85).- 28 (III/84).- 29 (XII/84).- 22 (II/85, IV/85).- 41 (II/85).- 54 (IX/84).- 86 (XI/84).- 5 (IV/85).- 100 (XI/81).

Rhopalodia musculus (Kützing) O. Müller

De $40 \times 15 \mu \mathrm{m}$, con 3 costillas y 14-16 estrías en $10 \mu \mathrm{m}$.

Bentónica en cursos de agua salobre o muy mineralizada (Tabla VI). Para Margalef (1954b) esta especie es más halófila que la anterior.

Loc.: 3 (IV/84, IX/84).- 5 (IV/85).- 41 (II/85).

Hantzschia amphyoxis (Ehrenberg) Grunow

De $50 \times 8-9 \mu \mathrm{m}$ con 20 estrías y 8 fíbulas cada $10 \mu \mathrm{m}$.

En charcas o cursos de agua alcalina dulce o salobre (Tabla VI). También sobre musgos en condiciones aerofíticas. Nuestras observaciones coinciden con las de Margalef (1954b) y difieren de las de Germain (1981).

Loc.: 26 (III/84, V/85).- 22 (II/83, II/85).- 117 (I/82).- 118 (XI/81).- 119 (XI/81).- 101 (IV/82).- 6 (IV/84).- 4 (IV/85).

Bacillaria paxillifer (Müller) Hendey

De 100 x $6 \mu \mathrm{m}$ con 6 fíbulas y 20-25 estrías cada $10 \mu \mathrm{m}$.

Bentónica en charcas o cursos de agua salobre o dulce. Soporta la polución (Tabla VI). Para Germain, (1981) se trata de una especie euroica.

Loc.: 4 (IV/84).- 11 (V/84).- 6 (IV/84).- 3 (IX/84).

Nitzschia acicularis W. Smith

De $75 \times 5 \mu \mathrm{m}$, con 16-20 fíbulas en $10 \mu \mathrm{m}$.

Sobre plantas sumergidas en cursos de agua alcalina dulce (Tabla VI).

Loc.: 26 (V/85).- 29 (XII/84).

Nitzschia acuta Hantzsch

De $95 \times 10 \mu \mathrm{m}$, con 6-7 fíbulas cada $10 \mu \mathrm{m}$.

Bentónica en cursos de agua alcalina dulce bastante mineralizada (Tabla VI).

Loc.: 31 (I/82).

Nitzschia amphibia Grunow

De $53 \times 9 \mu \mathrm{m}$, con 7-8 fíbulas y 16 estrías cada $10 \mu \mathrm{m}$.

Entre las plantas sumergidas de las riberas de cursos de agua dulce alcalina no polucionados (Tabla VI), aunque según Germain (1981) la polución no lo daña.

Loc.: 52 (III/81).

Nitzschia clausii Hantzsch

De $20-50$ x $4 \mu \mathrm{m}$, con 10-12 estrías cada $10 \mu \mathrm{m}$.

Sobre algas filamentosas en arroyos salinos junto con otras especies congéneres.

Germain (1981) la recolectó en aguas completamente dulces.

Loc.: 8 (VI/84).

Nitzschia closterium (Ehrenberg) W. Smith

De 40-150 x 2-4 $\mu \mathrm{m}$, con 12-17 fíbulas en $10 \mu \mathrm{m}$.

En cursos de agua salobre (Tabla VI), en relación con algas filamentosas.

Loc.: 6 (IV/84).- 5 (IV/84, IV/85).- 3 (IX/84, XI/84).- 4 (IV/84).- 86 (XI/84).

Nitzschia denticula Grunow

De 20 x $5 \mu \mathrm{m}$ con 6-7 costillas y 15-17 estrías en $10 \mu \mathrm{m}$.

Epífita sobre algas filamentosas en arroyos de agua salobre.

Loc.: 3 (IV/85).

Nitzschia dissipata (Kützing) Grunow

De $25 \times 4 \mu \mathrm{m}$, con 14 fíbulas en $10 \mu \mathrm{m}$. 
En el interior de tubos mucosos en las riberas de cursos de agua dulce o salobre. Soporta la polución si bien Germain (1981) opina lo contrario. (Tabla VI).

Loc.: 61 (VIII/83).- 62 (VII/83).- 3 (VII/84).- 1 (VII/83).- 4 (IV/84).- 8 (IX/84).

Nitzschia dubia W. Smith

De $115 \times 15 \mu \mathrm{m}$, con 10 fíbulas y $20-22$ estrías cada $10 \mu \mathrm{m}$.

En riberas de cursos de agua alcalina salobre o dulce. Soporta la polución orgánica (Tabla VI). Generalmente considerada como típicamente halófila, Germain (1981) también la encontró en agua totalmente dulce.

Loc.: 9 (IX/85).- 49 (VII/83).- 75 (VI/83).- 63 (VII/83).- 8 (VII/83, IX/84).- 28 (IX/85).- 26 (VIII/83).- 29 (XII/84).- 22 (II/83, IV/85).- 41 (II/85).- 3 (IV/84).- 7 (VII/83).

Nitzschia hungarica Grunow

De $40 \times 6 \mu \mathrm{m}$ con 12 fíbulas y $16-18$ estrías cada $10 \mu \mathrm{m}$.

Bentónica. Cursos de agua dulce muy mineralizada o salobre. Estas observaciones coinciden plenamente con las de Germain (1981).

Loc.: 3 (VII/84).- 22 (II/83).

Nitzschia obtusa W. Smith

De $300-335 \times 7 \mu \mathrm{m}$ con 5 fíbulas y 20 estrías cada $10 \mu \mathrm{m}$.

Junto con $V$. scalpelliformis Grunow habita los cursos de agua salobre o de muy elevada mineralización. Soporta polución (Tabla VI). Germain (1981) la recolectó en agua completamente dulce.

Loc.: 54 (VII/83).- 60 (VII/83).- 120 (VIII/83).- 121 (VIII/83).- 51 (VII/85).- 3 (IV/84, IX/84).- 79 (VII/83).- 53 (IV/84).- 6 (IV/84).- 5 (IV/84).- 4 (IV/84).- 8 (IX/84).- 7 (VII/83).- 31 (I/82).

Nitzschia cf. palea (Kützing) W. Smith

De 33 × $4 \mu \mathrm{m}$ con $10-12$ fíbulas y $30-40$ estrías cada $10 \mu \mathrm{m}$. VI).

Puede soportar gran variedad de condiciones y es muy resistente a la polución (Tabla (IX/85).

Loc.: 108 (IX/83).- 47 (IX/83).- 14 (VIII/85).- 76 (VI/83).- 26 (III/84, IX/85).- 40

\section{Nitzschia pusilla Kützing}

De $25 \times 5 \mu \mathrm{m}$ con 16 fíbulas en $10 \mu \mathrm{m}$.

Riberas de cursos de agua alcalina (Tabla VI).

Loc.: $26(\mathrm{~V} / 85)$.

Nitzschia recta Hantzsch

De $66 \times 7 \mu \mathrm{m}$ con $7-8$ fíbulas y 40 estrías cada $10 \mu \mathrm{m}$.

Sobre plantas sumergidas de las riberas de cursos de agua alcalina dulce (Tabla VI).

Loc.: 52 (III/81).

Nitzschia sigma (Kützing) W. Smith

De $150 \times 7 \mu \mathrm{m}$ con 11 fíbulas y $24-34$ estrías cada $10 \mu \mathrm{m}$.

Riberas de cursos de agua alcalina-salobre (Tabla VI), como ya indicaba Margalef (1954b). Para Germain (1981) se trata de una especie eurihalina.

Loc.; 4 (IV/84).- 3 (IV/84).- 5 (IV/84).- 8 (IX/84).

Nitzschia sigmoidea (Ehrenberg) W. Smith

De 350-385 x $10 \mu \mathrm{m}$, con 5-8 fíbulas y 25-30 estrías cada $10 \mu \mathrm{m}$.

Entre las plantas sumergidas de las riberas de cursos de agua alcalina dulce o salobre (Tabla VI). En Margalef (1954b) no se cita para aguas salobres.

Loc.: 88 (VII/83).- 26 (IX/84, IX/85).- 28 (III/84, IX/85).- 27 (IV/83, III/84, IX/85).29 (III/84, XII/84).- 22 (XI/84, II/85, IV/85).- 4 (III/84, IV/84).- 3 (XI/84).- 5 (IV/85).- 31 (I/82). 
Nitzschia tryblionella Hantzsch

Hemos recolectado la v. debilis (Arnott) A. Mayer de $18 \times 11 \mu \mathrm{m}, 10$ fíbulas y 12 estrías cada $10 \mu \mathrm{m}$ y v. subsalina Grunow de $40 \times 10 \mu \mathrm{m}$, con 5 fíbulas y 16 estrías cada $10 \mu \mathrm{m}$ en charcas o cursos de agua alcalina salobre o de elevada mineralización (Tabla VI).

Loc.: 22 (IX/84).- 4 (IV/84).- 6 (IV/84).- 34 (XI/81).

Clymatopleura elliptica (Brébisson) W. Smith

De $107 \times 64 \mu \mathrm{m}$ con 3-4 costillas en $10 \mu \mathrm{m}$.

Herpon de cursos de agua alcalina dulce (Tabla VII).

Loc.: 94 (VIII/85).- 26 (III/84, IX/85).- 28 (III/84, IX/85).- 29 (III/84).- 52 (XI/81).-

$101(\mathrm{IV} / 82)$.

Clymatopleura solea (Brébisson) W. Smith

De $100 \times 20 \mu \mathrm{m}$ con $7-8$ costillas en $10 \mu \mathrm{m}$.

En condiciones similares a la especie anterior aunque es más frecuente. Soporta la polución (Tabla VII).

Loc.: 122 (VIII/85).- 58 (VIII/83).- 11 (VIII/85).- 81 (VIII/85).- 124 (VIII/85).- 94 (VIII/85).- 84 (VII/83).- 33 (VI/82).- 88 (VII/83).- 26 (III/84, IX/85).- 28 (III/84).- 27 (IX/85).- 29 (III/84, XII/84).- 34 (XI/81).- 31 (V/81, I/82).- 33 (VI/82).- 20 (IV/84).

Surirella angustata Kützing

De $65 \times 13 \mu \mathrm{m}$ con $60-70$ costillas en $100 \mu \mathrm{m}$.

Cursos de agua alcalina dulce. Herpon (Tabla VII).

Loc.: 26 (IX/85).

Surirella ovalis Brébisson

De $70 \times 26 \mu \mathrm{m}$ con $30-40$ costillas en $100 \mu \mathrm{m}$ y $17-18$ estrías cada $10 \mu \mathrm{m}$.

Herpon de charcas y cursos de agua alcalina dulce o salobre (Tabla VII). Coincide con la ecología dada por Germain (1981).

Loc.: 12 (VII/83).- 104 (XII/82).- 75 (VI/83).- 51 (VIII/83).- 33 (IV/82).- 31 (I/82).- 26 (IX/84, IX/85).- 28 (III/84, IX/85).- 27 (IV/83).- 29 (III/84, XII/84).- 22 (IV/85).- 41 (II/85).- 53 (IV/84).- 4 (IV/84).- 6 (IV/84).- 3 (IX/84).- 8 (IX/84).- 86 (XI/84).- 32 (V/82, XI/81).- 34 (XI/81).- $30(\mathrm{~V} / 81)$.

\section{Surirella ovata Kützing}

De $27-45$ x $17-18 \mu \mathrm{m}$, con $60-80$ costillas en $100 \mu \mathrm{m}$ y $16-20$ estrías en $10 \mu \mathrm{m}$.

Cursos de agua alcalina dulce. Herpon (Tabla VII).

Loc.: 28 (III/84).- 34 (XI/81).- 32 (V/82).- 31 (L/82).- 69 (VI/81).

Surirella peisonis Pantocsek

De 80 × $60 \mu \mathrm{m}$, con $40-50$ costillas en $100 \mu \mathrm{m}$ y 14 estrías en $10 \mu \mathrm{m}$.

Herpon de charcas o cursos de agua de elevada mineralización o salobres (Tabla VII).

Loc.: 12 (VII/83).- 75 (VI/83).- 64 (VII/83).- 51 (VII/83).- 38 (VIII/85).- 31 (I/82).- 28 (II/84).- 29 (III/84).- 41 (II/85).- 7 (IX/840.- 54 (XI/840.- 86 (XI/84).

Surirella spiralis Kützing

De $135 \times 75 \mu \mathrm{m}$ con $15-30$ costillas en $100 \mu \mathrm{m}$.

Herpon de charcas muy someras o cursos de agua alcalina dulce muy mineralizada (Tabla VII).

Loc.: 40 (IX/85).- 26 (III/84).- 28 (III/84).- 29 (III/84).- 34 (X/81).- 31 (I/82).

Surirella striatula Turpin

De $108-140$ x $75-87 \mu \mathrm{m}$ con 12 costillas en $100 \mu \mathrm{m}$ y $12-20$ estrías en $10 \mu \mathrm{m}$.

Herpon de charcas someras o de cursos de agua salobre (Tabla VII).

Loc.: 64 (VII/83).- 7 (VII/83, IX/84).- 79 (VII/83).- 86 (XI/84).

Surirella subsalsa W. Smith

De $20-40$ × $8-10 \mu \mathrm{m}$ con 30 costillas en $100 \mu \mathrm{m}$ y 16 estrías en $10 \mu \mathrm{m}$.

Herpon de aguas remansadas de arroyos de agua salobre aunque Germain (1981) sólo la recolecta en el litoral. 
M. Aboal

Loc.: 4 (IV/85).

Surirella tenera Gregory v. nervosa A. Schmidt

De $140 \times 40 \mu \mathrm{m}$ con 21 costillas en $100 \mu \mathrm{m}$.

Herpon en charcas ribereñas de un curso de agua alcalina dulce (Tabla VII).

Loc.: 40 (IV/83).

Campylodiscus clypeus Ehrenberg y su v. biscostata W. Smith

De $110-120 \mu \mathrm{m}$ de diámetro con 19-20 costillas cada $100 \mu \mathrm{m}$.

Herpon en cursos de agua alcalina salobre (Tabla VII).

Loc.: 64 (VII/83).- 7 (VII/83, IX/84).- 86 (XI/84).

Campylodiscus noricus Ehrenberg v. hibernica (Ehrenberg) Grunow

De 45-64 $\mu \mathrm{m}$ de diámetro, con 10-20 costillas en $100 \mu \mathrm{m}$.

Herpon de charcas o cursos de agua alcalina dulce o salobre. (Tabla VII).

Loc.: 20 (VIII/85).- 64 (VII/83).- 26 (III/84, IX/85).- 28 (III/84).- 3 (IV/84).- 32 (XI/81).- 31 (V/81, I/82).- 34 (XI/81).- 86 (XI/84).

\section{DIS C USION}

Las comunidades de Diatomeas y las algales en general recolectadas en la zona del sudeste español, fundamentalmente en la Cuenca del Segura se distribuyen en función de las oscilaciones del nivel del agua y de los cambios de calidad físico-química del agua que provocan el substrato y la influencia humana (Aboal, 1987).

El tipo de comunidad más extendido en la cuenca es el que Margalef (1951) define como característica de la montaña media caliza. Sólo en el nacimiento del río Segura se recolectan especies como; Diatoma hiemale v. mesodon, Meridion circulare y Cymbella ventricosa indicadoras de una altitud media-alta y de una calidad del agua óptima (Fjerdingstad, 1965).

Se pueden diferenciar dos grandes grupos de cursos de agua en la zona: los que poseen aguas alcalinas dulces y las que las tienen alcalino-salobres. Las primeras son mayoritarias en extensión y albergan el número más grande de especies, que ocupan los biótopos epilíticos y epifíticos, y con una composición comparable a la de otros ríos de condiciones similares: Descy (1976), Sabater (1987), Leclercq \& Maquet (1987), Cazaubon \& Orsini (1988), Holmes \& Whitton (1981).

Los arroyos de aguas salobres sólo se desarrollan en las zonas en que predominan las margas del Keuper; las comunidades que dominan estos ambientes son muy similares a las que se pueden encontrar en las lagunas litorales: Pleurosira laevis, Chaetoceros wighamii, Diploneis didyma... (Aboal, 1988).

En ambos casos se producen situaciones de aguas polucionadas que traen consigo una drástica reducción de las poblaciones. Entre las especies más resistentes están Nitzschia palea y Cyclotella meneghiana, de modo similar a lo que ocurre en el río Ter (Sabater, 1987).

\section{BIBLIOGR A F I A}

ABOAL, M. -1987- Flora algal epicontinental de la cuenca del río Segura, S.E. de España. Tesis Doctoral. Fac. Biología. Univ. Murcia. 
ABOAL, M. -1988- Diatomées des cours d'eau saumâtres temporaires ou permanentes du S.E. de l'Espagne. Bull. Soc. Roy, Bel. n 10:48-54.

AVILES, ET AL. -1973-Reconocimiento limnológico de la Cuenca del Segura. Centro de Estudios Hidrográficos MOPU. Madrid.

AVILES, ET AL. -1980-Reconocimiento limnológico del Segura II fase. Centro de Estudios Hidrográficos. MOPU. Madrid.

BOLD, H.C. \& WYNNE, M.J. -1985-Introduction to the algae. Prentice Hall. New Jersey.

CAZAUBON, A. \& ORSINI, A. -1988- Influence du couvert végétal sur les communautés diatomiques épilithiques de deux rivières de Corse (Mediterranée). Bull. Soc. Roy. Bot. Belg. 10:35-47.

COMPERE, P. -1982- Taxonomic Revision of the Diatom Genus Pleurosira (Eupodiscaceae), Bacillaria 5:165-190.

DESCY, J.P. -1976- La végétation algale benthique de la Somme (France) et ses relations avec la qualité des eaux. Mém. Soc. Roy. Bot. Belg. 7:101-128.

FJERDINGSTAD, E. -1965- Taxonomy and saprobic valency of benthic phytomicroorganisms. Int. revue ges. Hydrobiol. 50:475-604.

GERMAIN, F. -1981- Flore des Diatomées (Diatomophycées) eaux douces et saumâtres du Massif Armoricain et des contrées voisines d'Europe occidentale. Societé Nouvelle des Editions Boubée. Paris.

HOLMES, N.T.H. \& WHITTON, B.A. -1981- Phytobenthos of the river Tees and its tributaries. Freshwater Biology 11:139-163.

HUSTEDT, F. -1930/1966-Die kieselalgen Deutschlands, Österreich und der Schweiz unter Berücksichtigung der übrigen Länder Europas Sowie der angrenzenden Meeresgebiete. Teil 1, 2, 3. Otto Koeltz Scientific Publishers.

HUSTEDT, F. -1930- Die Süsswasser-Flora Mitteleuropa. Bacillariophyta (Diatomeae). Verlag von Gustav Fisher.

KRAMMER, K. \& LANGE-BERTALOT, H. -1986- Bacillariophyceae. 1. Naviculaceae. Süsswasserflora von Mitteleuropa 2/1. Gustav Fisher Verlag. Jena.

KRAMMER, K. \& LANGE-BERTALOT, H. -1988- Bacillariophyceae. 2. Bacillariaceae, Epithemiaceae, Surirellaceae. Süsswasserflora von Mitteleuropa 2/2. Gustav Fisher Verlag. Jena.

LANGE-BERTALOT, H. -1980- Zur systematischen Bewertung der band-förmigen kolonien bei Navicula und Fragilaria. Nova Hedwigia XXXIII:723-787.

LECLERCQ, L. \& MAQUET, B. -1987-Deux nouveaux indices chimiques et diatomiques de qualité d'eau courante. Institut Royal des Sciences Naturelles de Belgique.

MARGALEF, R. -1951- Regiones limnológicas de Cataluña y ensayo de sistematización de las asociaciones de algas. Collect. Botanica 3(1):43-67.

MARGALEF, R. -1954a- Materiales para una flora de las algas del N,E. de España Va, Bacillariophyta. Coll. Bot. vol. IV. fasc. I. $n^{2}$ 4: 53-79.

MARGALEF, R. -1954b-Materiales para una flora de las algas del N.E. de España. Vb, Bacillariophyta. Coll. Bot. vol. IV. fasc. II, $\mathrm{n}^{0} 12:$ 183-201.

MARGAlEF, R., PLANAS, M.D., ARMENGOL, J., VIDAL, A., PRAT, N., GUISET, A., TOJA, J. \& ESTRADA, M. -1977- Limnología de los embalses españoles. Dirección General de Obras Públicas. Centro de Estudios Hidrográficos.

SABATER, S. -1987-Estudi de les poblacions d'algues del riu Ter. Tesis Doctoral. Facultad de Biología. Universidad Central de Barcelona.

SCHOEMAN, F.R. \& ARCHIBALD, R.E.M. -1986- Observations on Amphora species (Bacillariophyceae) in the British Museum (Natural History). V. Some species of the subgenus Amphora. S. Afr. I. Bot. 52(5):425-437.

TOMAS, X. -1979- Diatomeas de las aguas epicontinentales de España: géneros Cymbella y Gomphonema. Tesina de Licenciatura. Fac. Biología. Univ. Central de Barcelona.

TOMAS, X. -1981- El género Mastogloia en los sistemas acuáticos del litoral mediterráneo español. Coll. Bot. vol. 13(2):929-944. 
Tabla I: Localidades

1.- Fuente de la Plata. Mesones. Albacete. WH 5960. 2.- Azarbe Riacho. El Hondo. Alicante. 3.- Rambla del Salar. Ulea. Murcia. XH 5125. 4.- Rambla del Carrizalejo. Murcia. XH 5326. 5.- Rambla del Tinajón después del vertido. Murcia. XH 5021. 6.- Barranco del Mulo. 2. Murcia. XH 5126. 7.- Rambla del Salar. Nacimiento. Blanca. Murcia. XH 4730. 8.- Rambla del Salar después del vertido de Blanca. Murcia. XH 4928. 9.- Río Segura antes de Calasparra. Murcia. XH 1534. 10.- Río Segura después de Almadenes. Murcia. XH 6340. 11.- Río Segura. Archena. Murcia. XH 4920. 12.- Rambla del Judío. Cieza Murcia. XH 3749. 13.- Río Segura después del vertido de Molina. Murcia. XH 5612. 14.- Río Segura antes de Alquerías. Murcia. XH 7209. 15.- Arroyo de Morote. Albacete. XH 6652. 16.- Arroyo de la Vega. Albacete. XH 5161. 17.- Arroyo Salado. Riópar. Albacete. WH 5260. 18.- Río Mundo. El Laminador. Albacete. WH 5551. 19.- Río Mundo. La Alfera. Albacete. WH 6262. 20.- Río de Las Hoyas. El Encebrico. Albacete. WH 5369. 21.- Río Mundo. Ayna. Albacete. WH 8067. 22.- Rambla del Puerto. El Portazgo. Murcia. XG 6198. 23.- Río Madera. Nacimiento. Jaén. WH 3436. 24.- Río Tus. Fábricas de Madera. Albacete. WH 4044. 25.- Río Benamor. Nacimiento. Murcia. WH 7330. 26.- Río Benamor. La Puerta. Moratalla. Murcia. WH 9130. 27.- Río Benamor. Cenajo del Agua Cernía. Murcia. WH 8829. 28.- Río Benamor antes de Calasparra. Murcia. XH 0530. 29.- Río Benamor. Casas de Pelota. Murcia. XH 0030. 30.- El Aceniche. Bullas. Murcia. XH 1305. 31.Río Pliego. Meseta de la Plata. Murcia. XH 3510. 32.- Rambla Salada. Zarcilla de Ramos. Murcia. WG 9687. 33:- Fuente próxima al Salto Lucero. Bullas. Murcia. XH 1709. 34.- Río Quípar. Cehegín. Murcia. XH 0715. 35.- Río de la Fuente del Roble. Albacete. WH 5873. 36.- Río de las Acequias. Paterna de Madra. Albacete. WH 5673. 37.- Río Segura. Formentera. Alicante. XH 9717. 38.- Nacimiento del Turilla. Murcia. XH 0094. 39.- Río Guadalentín antes de Lorca. Murcia. XG 1471. 40.- Río Benamor. Somogil. Murcia. WH 9030. 41.- Rambla del Puerto cerca del Nacimiento. Murcia.XG 6294. 42.- Río Segura. Cieza. Murcia. XH 3743. 43.- Rambla del Moro. Murcia. XH 4332. 44.- Rambla del Mayés. Murcia. XH 4420. 45.- Río Segura. Baños de Archena. Murcia. XH 4921. 46.- Río Segura antes del Puente Lorquí-Ceutí. Murcia. XH 5217. 47.- Río Segura. FICA. Murcia. XH 6305. 48.Río Segura. El Raal. Alicante. XH 7412. 49.- Río Segura. Rojales. Alicante. XH 9918. 50. Arroyo de La Celada. Albacete. WH 5660. 51.- Rambla de Tobarra. Agramón. Albacete. XH 1952. 52.- Río Mula. Salto Lucero. Bullas. Murcia. XH 1709. 53.- Barranco del Mulo. 1. Murcia. XH 5127. 54.- Arroyo del Tus. Tus. Albacete. WH 4951. 56.- Nacimiento del Segura. Pontones. Jaén. WH 2671. 57.- Arroyo Madera. Jaén. WH 5039. 58.- Río Segura. Las Minas. Murcia. XH 1444. 59.- Río Segura después de la desembocadura del Benamor. Murcia. XH 1436. 60.- Rambla del Judío. Desembocadura. Murcia. XH 3645. 61.- Río Segura entre Guadarmar y Rojales. Alicante. YH 0019. 62.- Chorros del Mundo. Albacete. WH 4956. 63.- Fuente del Hueso. Mora de Santa Quiteria. Albacete. XH 2366. 64.- Rambla de Tobarra. Minateda. Albacete. XH 2160. 65.- Río Mula. Rambla Perea. Murcia. XH 3115. 66.- Río Endrinales. Albacete. WH 5166. 67.- Río Viñazos. Albacete. WH 5470. 68.- Río de la Hoyas. Batán del Puerto. Albacete. WH 5670. 69.- Río Mula. Ucenda. Murcia. XH 1509. 70.- Fuente camino de Yetas. Albacete. XH 6129. 71.- Río Mundo entre Alfera y Alejos. Albacete. WH 6563. 72.- Río Segura. Camino de la Graya. Albacete. WH 5339. 73.- Arroyo de las Fuentes. Nerpio. Albacete. WH 6122. 74.- Río Segura. Desembocadura. Alicante. YH 0419. 75.- Arroyo de Haches. Albacete. WH 7375. 76.Arroyo de Potiche. Albacete. WH 7270. 77.- Barranco de Ubricas. Albacete. WH 9031. 78.- Arroyo de Hondares. Murcia. WH 9030. 79.- Rambla de Cantalar. Murcia. XH 6426. 80.- Río Segura antes del Argos. Murcia. XH 1532. 81.- Río Segura. Llano de Molina. Murcia. XH 5415. 82.- Río Segura. Alguazas. Murcia. XH 5513. 83.- Río Segura. Contraparada. Murcia. XH 5607. 84.- Arroyo del Gollizo. Riópar. Albacete. XH 5162. 85.Fuente de Isso. Albacete. XH 0862. 86.- Rambla de Pilarico. Murcia. XH 4823. 87.Cañadas de Nerpio. Albacete. WH 5315. 88.- Río Tus. La Rala. Albacete. WH 5951. 89.Río Mencal. Albacete. WH 5974. 90.- Río Segura antes del embalse de Anchuricas. Jaén. WH 4128. 91.- Arroyo de la Sierra. Vados de Tus. Albacete. WH 4948. 92.- Río Tus en los Baños. Albacete. WH 4245. 93.- Arroyo Taibilla. 1. Albacete. WH 5521. 94.- Río Taibilla. El Peñón. Albacete. WH 6426. 95.- Río Taibilla. La Toba. Albacete. WH 6331. 96.- 
cete. WH 6262. 97.- Fuente del Marqués. Murcia. WH 9817. 98.- Barranco de Galán. Calasparra. Murcia. WH 3524. 99.- Río Tus antes de Los Baños. Albacete. WH 4245. 100.Embalse de Puentes. Lorca. Murcia. XG 0477. 101.- El Carrascalejo. Bullas. Murcia. XH 1313. 102.- Río Mula. Campos del Río. Murcia. XH 4511. 103.- Río Turilla. Zarcilla de Ramos. Murcia. XH 0090. 104.- Río Madera. Cruce de Pontones. Jaén. WH 3332. 105.Río Segura después desembocadura del Madera. Jaén. WH 3524. 106.- Arroyo Romagillos. Albacete. WH 3230. 107.- Río Segura después de la desembocadura del Mula. WH 5611. 108.- Rambla de Ambroz. Blanca. Murcia. XH 4025. 109.- Río Taibilla. Las Claras. Albacete. WH 6943. 110.- Arroyo de la Fuente del Roble antes del río Acequias. WH 5671. 111.- Río Mundo. Las Hoyas. Albacete. WH 7767. 112.- Nacimiento del Benamor. Murcia. WH 7330. 112.- Río Segura. Embalse de la Fuensanta. Murcia. WH 6850. 113.- Embalse del Turrilla. Albacete. WH 6331. 114.- Arroyo de La Dehesa. Jaén. WH 7241. 115.- Río Madera (Mundo). Albacete. WH 6172. 116.- Fuente en la carretera Bogarra-Paterna de Madera. Albacete. WH 6573. 117.- Pico de la Selva. Bullas. Murcia. XH 1598. 118.- Río Argos. Caravaca. Murcia. WH 9916. 119.- Venta del Pino. Bullas. Murcia. XH 1006. 120.Presa de Guadarmar. Alicante. YH 0418. 121.- Río Segura cerca de Guadarmar. Alicante. YH 0521. 122.- Río Segura. Pontones. Jaén. WH 2919. 123.- Río Segura después del vertido de Molina. Murcia. XH 5612.

(Aceptado para su publicación el 15 de enero de 1989)

Dirección de la autora: Departamento de Biología Vegetal. Unidad Docente de Botánica. Facultad de Biología. Universidad de Murcia. 30070. Murcia. 


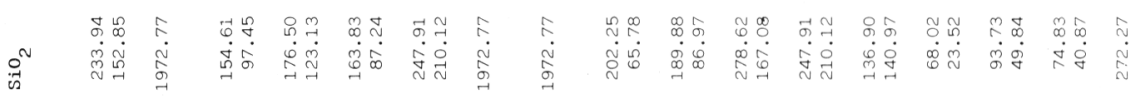

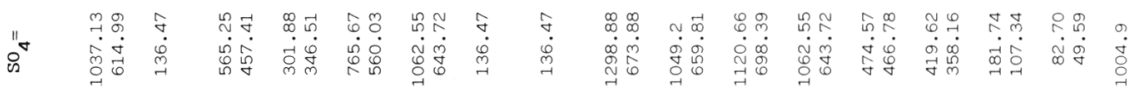

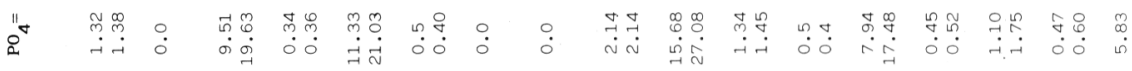

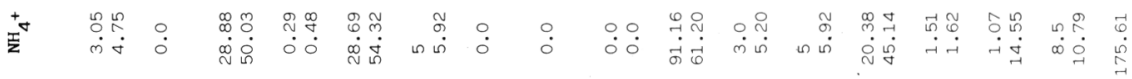

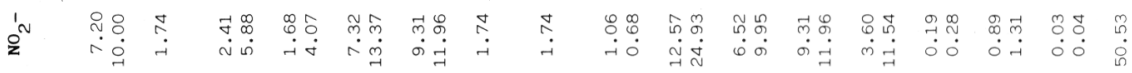

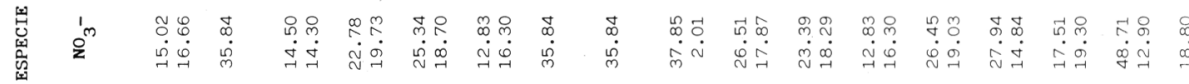

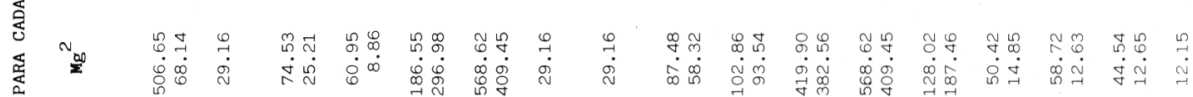

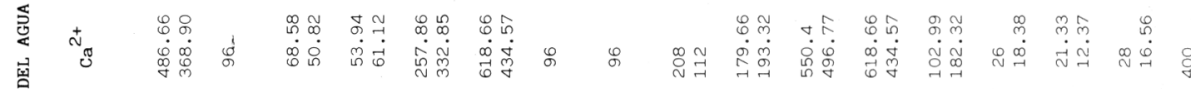

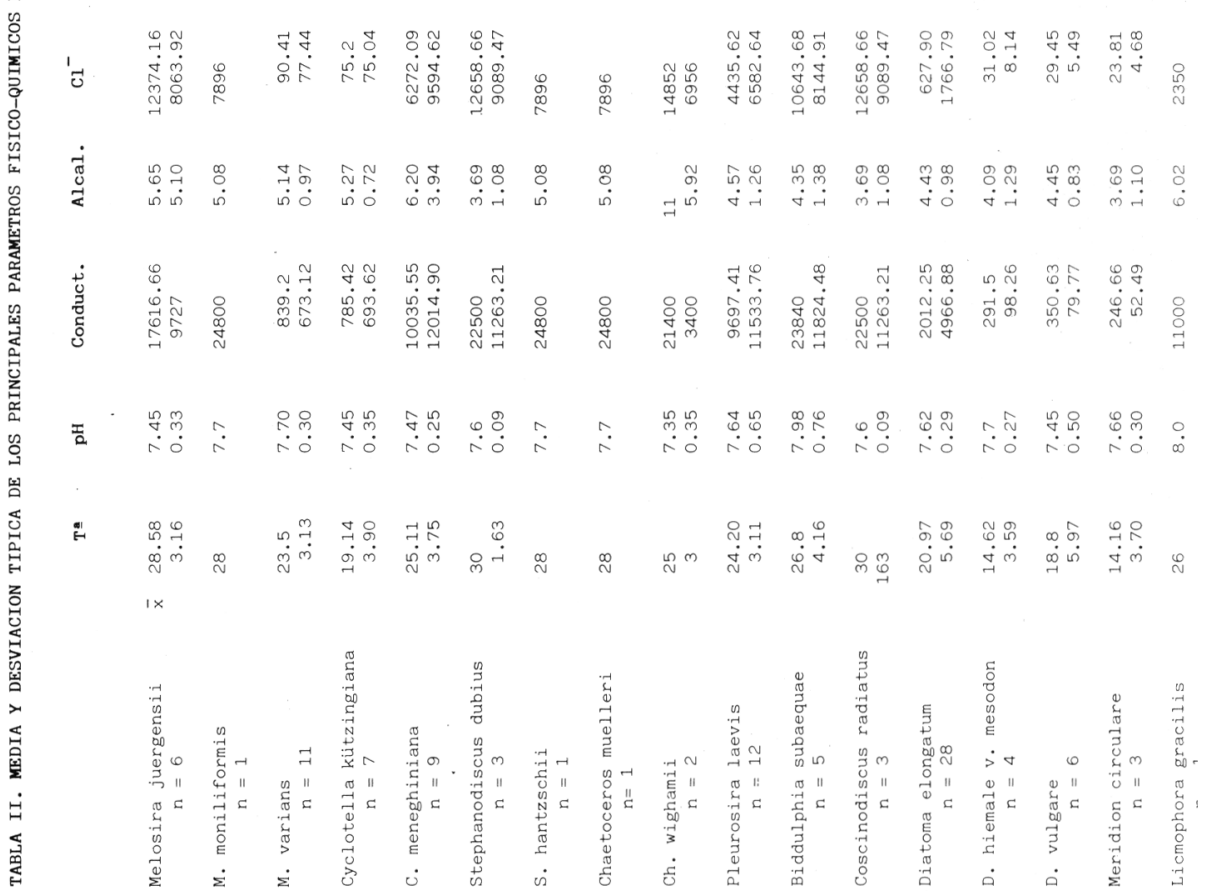


ง

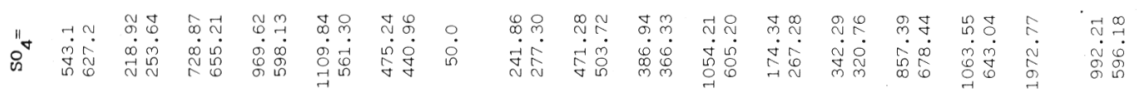

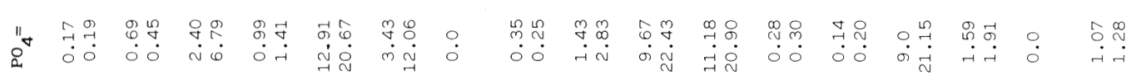

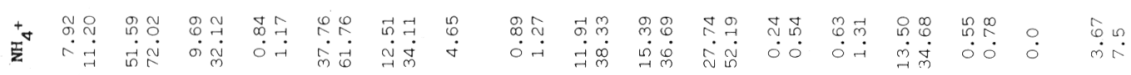

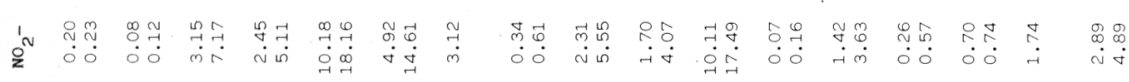

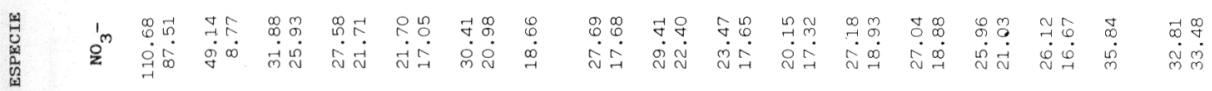

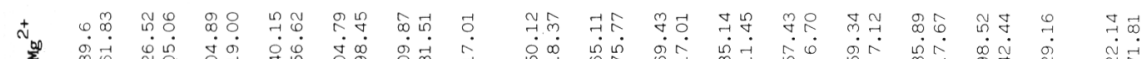

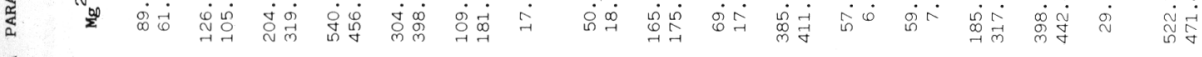

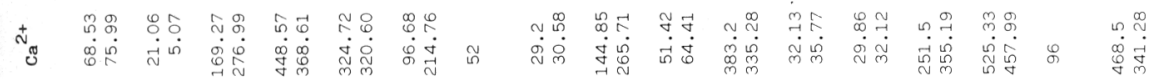

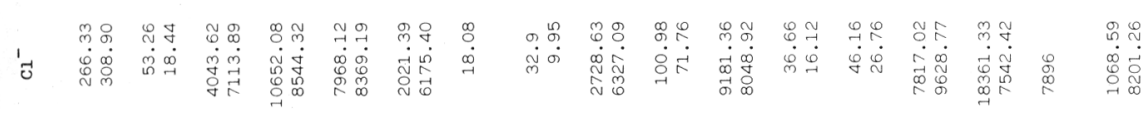

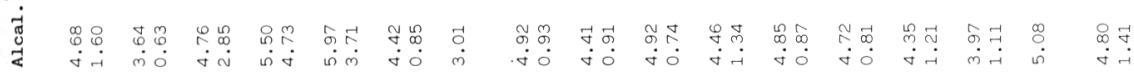

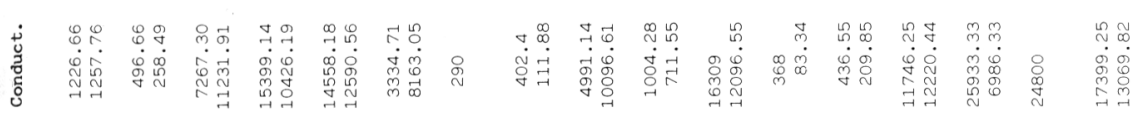
I.

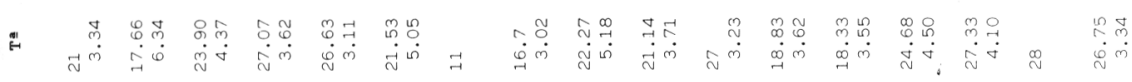
$1 \times$

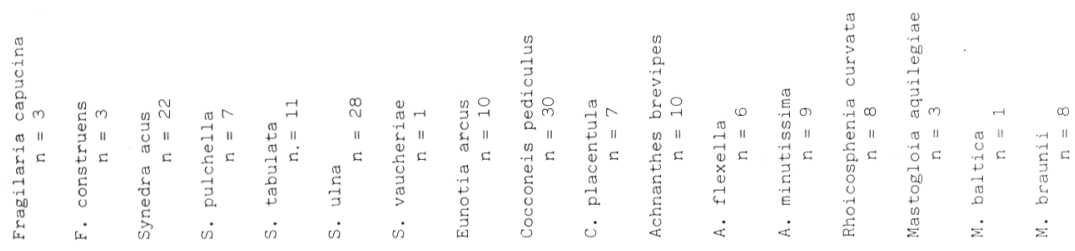


N 光 N

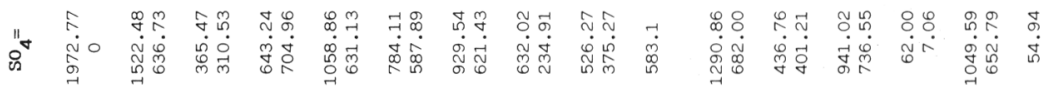

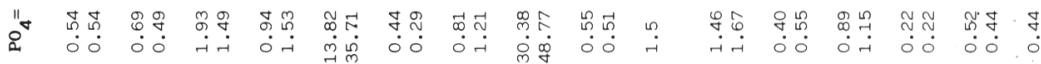

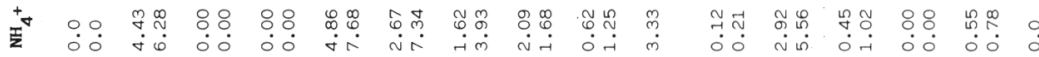

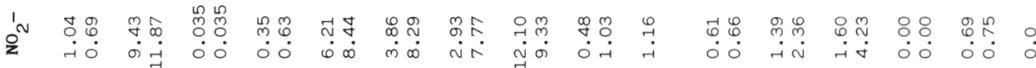

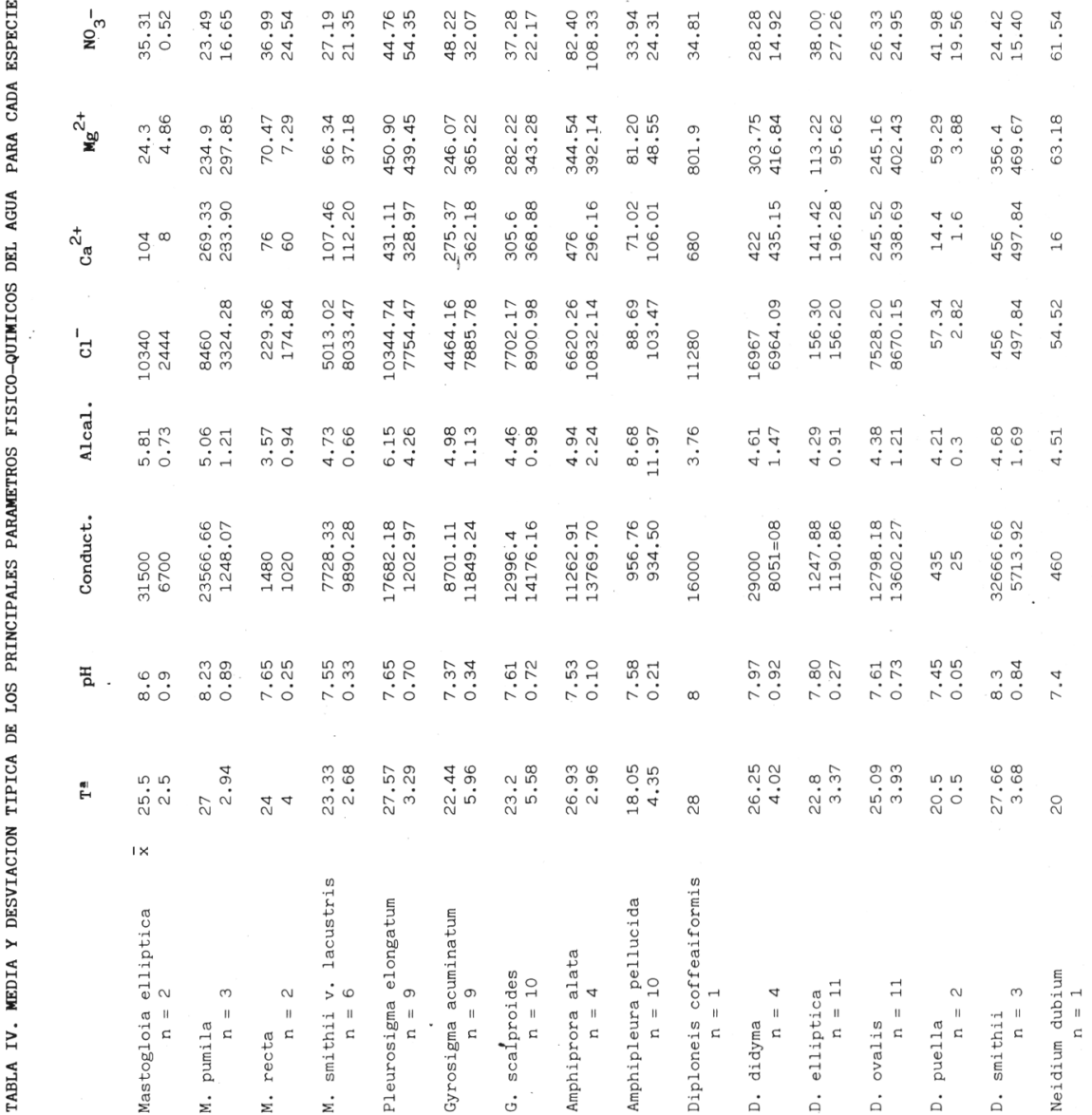




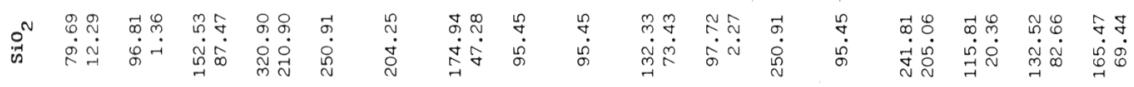

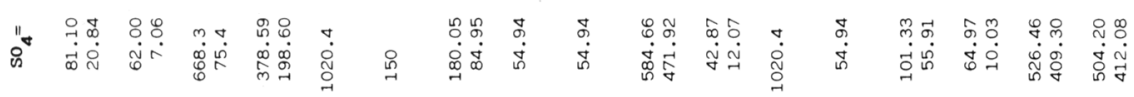

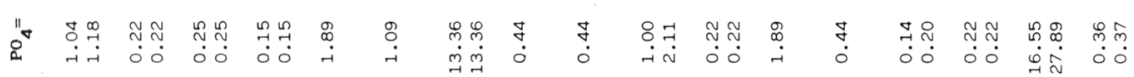

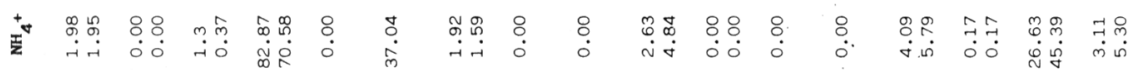

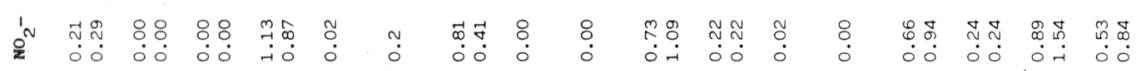

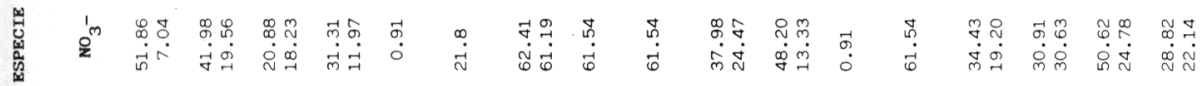

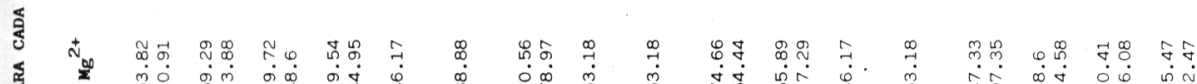

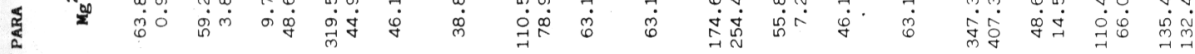

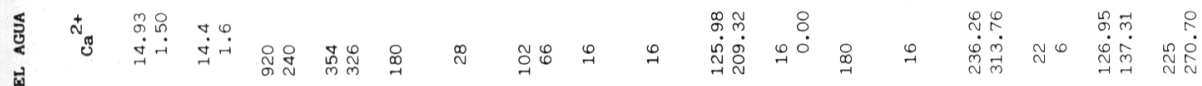

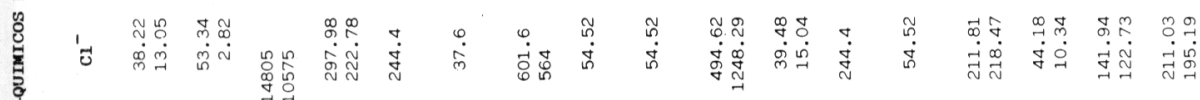

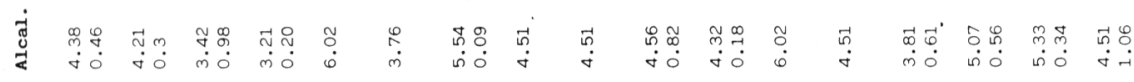

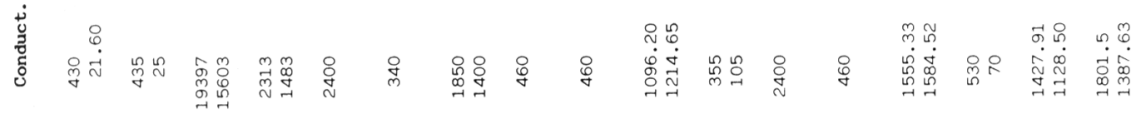

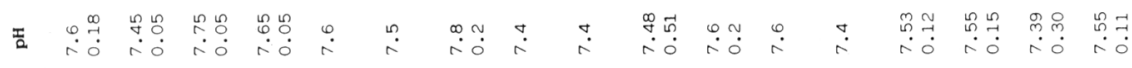

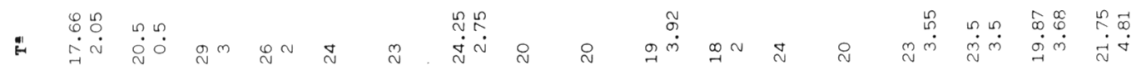
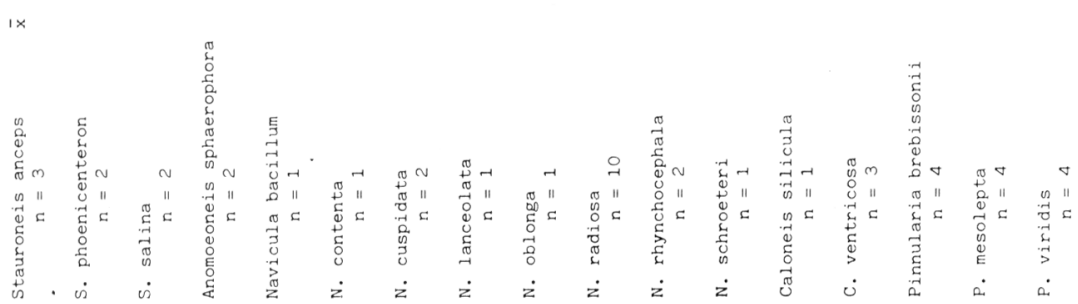


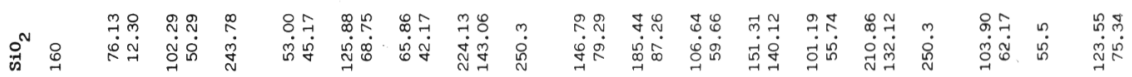

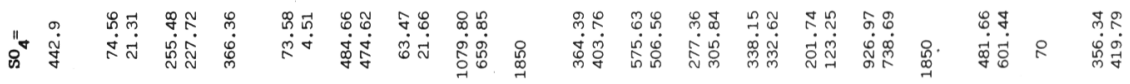

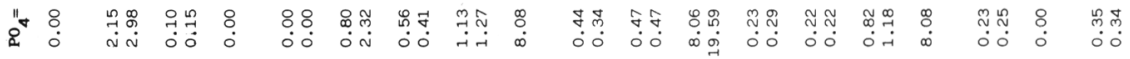

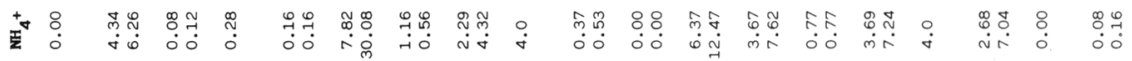

党

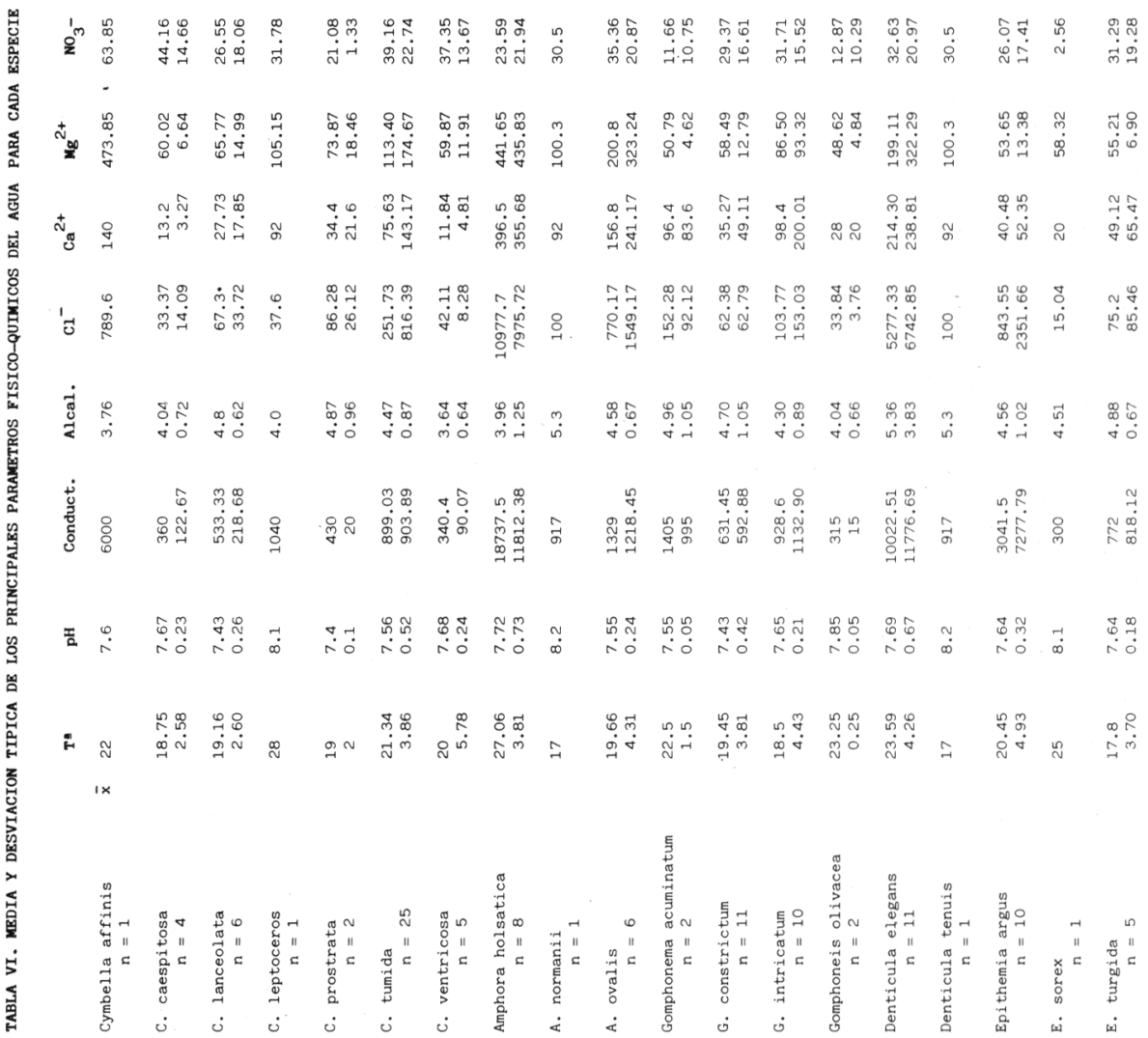




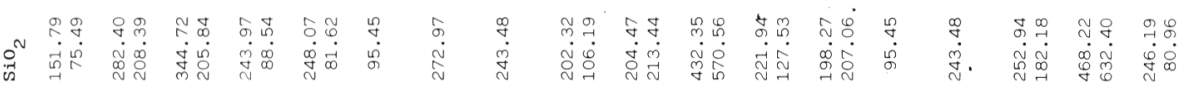
I

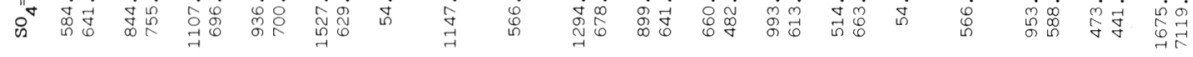

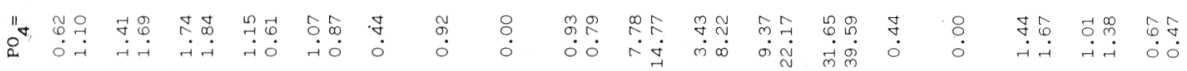

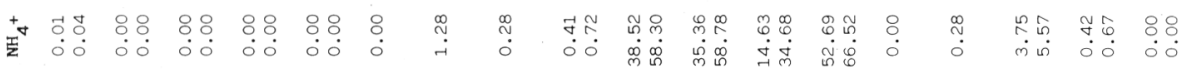

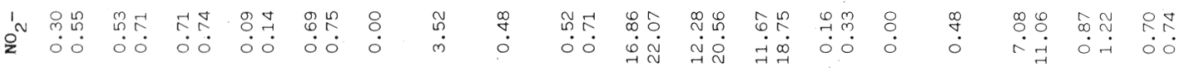

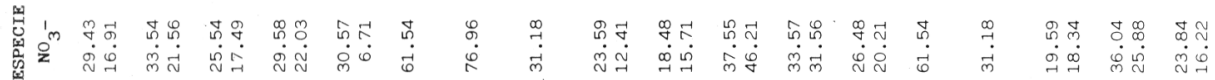
这

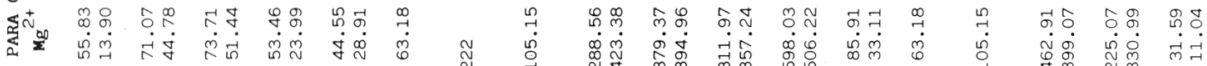
羌 畗金

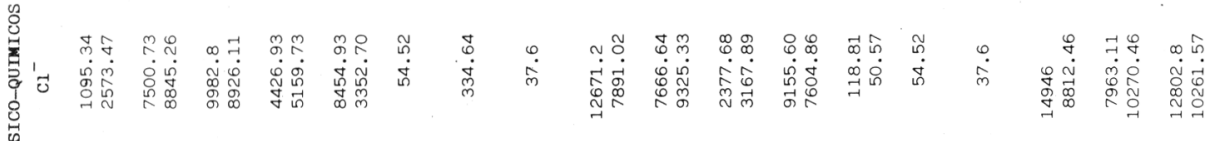

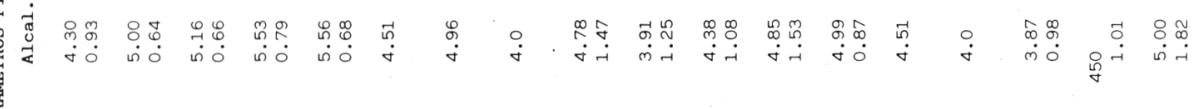

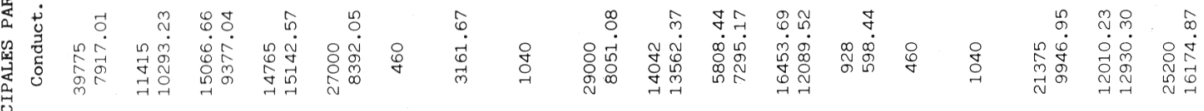

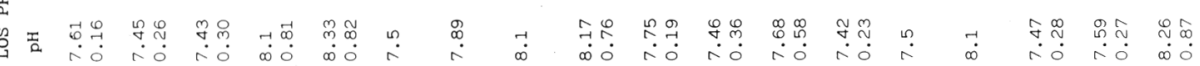
台

总:

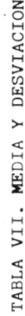

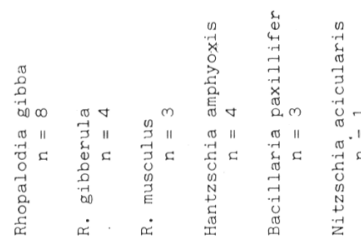

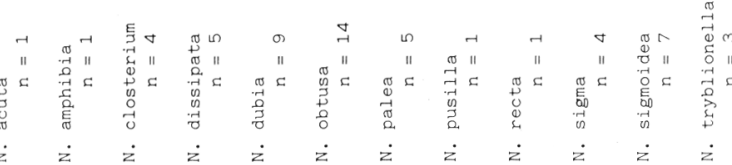




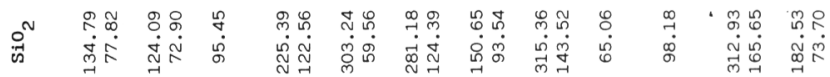

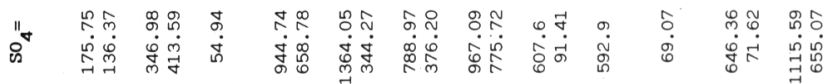

¿

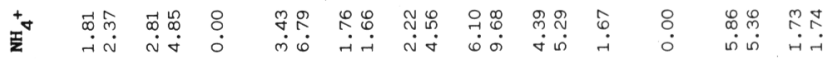

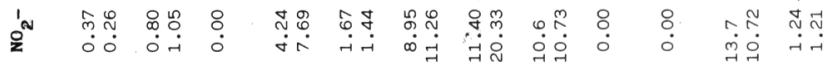

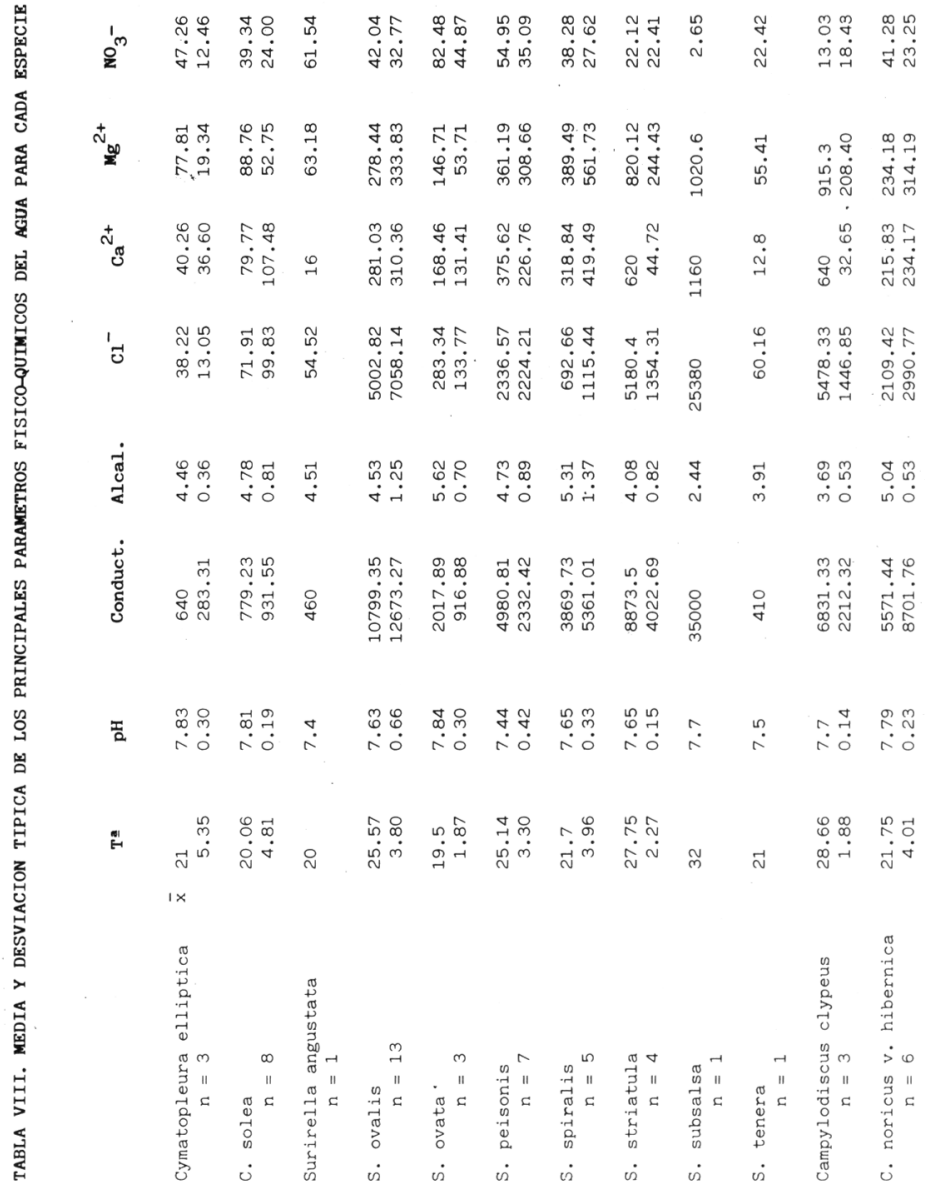

